\title{
DIVERSITY AND DISTRIBUTION OF TARDIGRADES (BILATERIA, TARDIGRADA) FROM THE IBERIAN PENINSULA, BALEARIC ISLANDS AND CHAFARINAS ISLANDS
}

\author{
N. Guil *
}

\begin{abstract}
Bibliographical information on the Tardigrada from Iberian Peninsula, Balearic Islands and Chafarinas Islands is compiled herein. 118 species are listed from the studied area. The first records of six Tardigrada species, Dactylobiotus parthenogeneticus, Dactylobiotus selenicus, Murrayon dianeae, Diphascon (Diphascon) nodulosum (first time in Europe), Diphascon (Diphascon) pingue and Isohypsibius marcellinoi that had not previously been found in the area are included. Bibliography data are analysed. After the analysis, it is concluded that more intensive, extensive and within more diverse habitats sampling effort should significantly increase the knowledge of tardigrade diversity in the studied area.

Keywords: Tardigrada, corology, Iberian Peninsula, Balearic Islands, Chafarinas Islands, new records, Apochela, Parachela, Arthrotardigrada, Milnesiidae, Calohypsibiidae, Hypsbiidae, Batillipedidae, Echiniscidae, Echiniscoididae.
\end{abstract}

\section{RESUMEN}

\section{Diversidad y distribución de las especies del phylum Tardigrada en la Península Ibérica, Islas Baleares e Islas Chafarinas}

En este artículo hemos recopilado la información existente en la bibliografía acerca del phylum Tardigrada en la Península Ibérica, Islas Baleares e Islas Chafarinas. La lista de especies del phylum Tardigrada incluye 118 especies en el área de estudio. Además, incluimos seis especies encontradas por primera vez en el área de estudio: Dactylobiotus parthenogeneticus, Dactylobiotus selenicus, Murrayon dianeae, Diphascon (Diphascon) nodulosum (por primera vez encontrado en Europa), Diphascon (Diphascon) pingue e Isohypsibius marcellinoi. En base al análisis realizado con los datos recopilados de la bibliografía, podemos concluir que un pequeño aumento en el esfuerzo de muestreo (intensivo, extensivo y aumentando la diversidad de hábitats recolectados) se traduce en un aumento significativo de la diversidad del phylum en el área estudiada.

Palabras clave: Tardigrada, corología, Península Ibérica, Islas Baleares, Islas Chafarinas, nuevas citas, Apochela, Parachela, Arthrotardigrada, Milnesiidae, Calohypsibiidae, Hypsbiidae, Batillipedidae, Echiniscidae, Echiniscoididae.

* Department of Biodiversity and Evolutive Biology. Museo Nacional de Ciencias Naturales de Madrid (CSIC). C/ José Gutiérrez Abascal, 2. 28006 - Madrid - Spain. Phone: +34.91.561.86.00 (Ext. 1215 / 1111).

E-mail: monng523@mncn.csic.es 


\section{Introduction}

Tardigrades are micrometazoans that can be found world-wide (McInnes, 1994; Nelson \& Marley, 2000), in many types of habitats (aquatic and terrestrial) within a film of water (Ramazzotti \& Maucci, 1983; Nelson, 1995). They are specially interesting for their capacity to enter in criptobiosis (Crowe, 1975; Nelson, 1995), with posible future applications in biomedicine (Crowe \& Crowe, 2000). Another important point is that the phylogenetic position of the Tardigrada gives rise to controversy, depending on whether morphological characters (Nelson, 1982; Nielsen, 1995) or molecular characters (Garey et al., 1996; Giribet et al., 1996; Garey et al., 1999) are studied. Nowadays, it seems that preserving genetic dissimilarity, based on phylogenetic information, is often of a higher conservation priority (Humphries et al., 1995; Hartvingsen, 2001; Swingland, 2001). Although conservation based on ecosystem and landscape approaches might be better, a singlespecies approach may still be appropriate as a first order conservation tool (Kieser, 1993). Therefore, we need the studied group's complete taxonomical information, up-to-date check lists, species diversity studies, relative abundances, corology, etc. to define the phylogeny robustly and, from that, to infer its biodiversity. All of this information, and even more basic information, are incomplete in the Tardigrada phylum.
Tardigrade species description, as it is the case with many other invertebrate groups, accelerated during XX century, along with the growth in the number of researchers involved in its investigation. Tardigrade researches in Iberian Peninsula began in 1911 (Madrid Moreno, 1911), 138 years after the discovery of Tardigrada phylum in 1773 (Goeze, 1773). In the Iberian Peninsula, tardigrade studies are mainly one-off descriptions of new taxa, while in other countries tardigrade researchers work other than with the description of new taxa, with the biology and the ecology of the group.

The main objective of the present work is to compile and up-date the entire body of tardigrade information (species diversity, corology, auteco$\operatorname{logy}$ ) for the Iberian Peninsula, the Balearic Islands and Chafarinas Islands, and to show how a small sampling effort (in many types of habitats) could greatly advance the knowledge of Iberian tardigrades, as one may infer from the bibliographic data.

\section{Material and Methods}

This work is based on taxonomic and geographic distribution information found in 34 scientific articles (Table I) on tardigrade species found in the Iberian Peninsula, the Balearic Islands and Chafarinas Islands (first article related with the area is Heinis, 1908, on Canary Island tardigrades), dated from 1911 (plankton from ponds in Madrid and

Table I.- - List of all the articles dealing with tardigrade information that have been published for Iberian Peninsula, Balearic Islands and Chafarinas Islands chronologically ordered. *1= Paper of Heinis, 1908 is not about Iberian Peninsula Tardigrada fauna, it is from Canarian Islands, but it is the first paper related to the area, $* 2=$ articles that deal with marine tardigrades from studied area.

Tabla I. - Lista de los artículos sobre los tardígrados en la Península Ibérica, Islas Baleares e Islas Chafarinas. $* 1=$ El artículo de Heinis, 1908, no es sobre los tardígrados de la Península Ibérica, es sobre los tardígrados de las Islas Canarias, pero es el primer artículo relacionado con el área a estudio, ${ }^{*} 2=$ artículos sobre los tardígrados marinos del área a estudio.

\begin{tabular}{lll}
\hline & \multicolumn{1}{c}{ ARTICLES } \\
\hline Heinis, 1908*1 & Rodríguez Roda, 1947*2 & Maucci, 1983 \\
Madrid Moreno, 1911 & Da Cunha, 1948 & Ramazzotti \& Maucci, 1983 \\
Pardo García, 1919 & Rodríguez Roda, 1949 & Lewin Osorio, 1984 \\
Pardo García, 1921 & Rodríguez Roda, 1951 & Maucci \& Durante Pasa, 1984 \\
Barros \& Da Cunha, 1937 & Rodríguez Roda, 1952 & Maucci \& Durante Pasa, 1985 \\
Da Cunha, 1941 & Mihelcic, 1954 & Maucci, 1991 \\
Da Cunha, 1943 & Mihelcic, 1955 & McInnes, 1991 \\
Da Cunha, 1944 a & Maucci, 1979 & Villora Moreno, 1993*2 \\
Da Cunha, 1944 b & Kristensen \& Hallas, 1980*2 & Villora Moreno \& De Zio Grimaldi, 1993*2 \\
Rodríguez Roda, 1946 & Fontoura, 1981 & De Zio Grimaldi \& Villora Moreno, 1996*2 \\
Da Cunha, 1947 a & Maucci \& Ramazzotti, 1981 & Villora Moreno \& De Zio Grimaldi, 1996*2 \\
Da Cunha, 1947 b & Fontoura, 1982 & \\
\hline
\end{tabular}




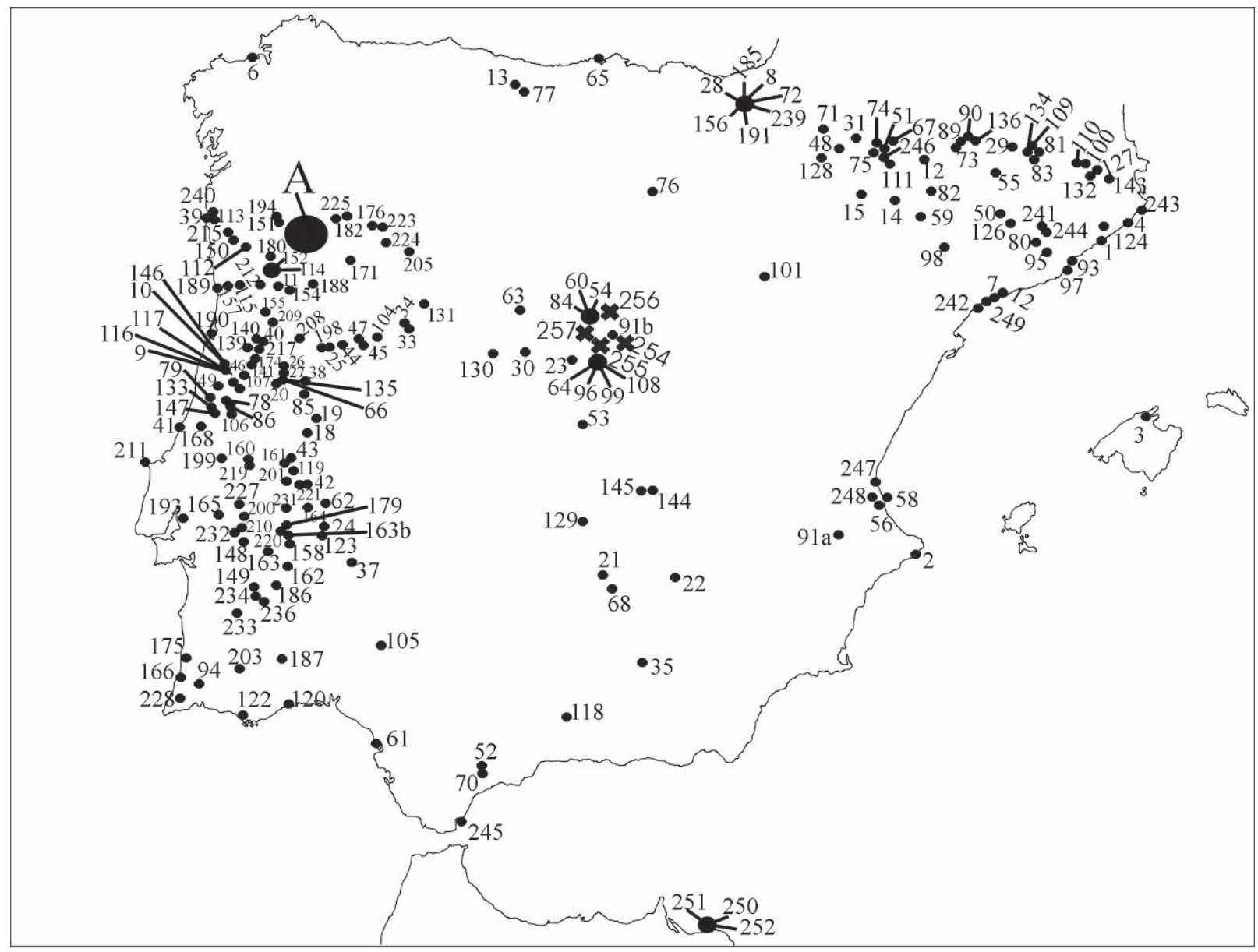

Fig. 1. - Localities sampled in the Iberian Peninsula, the Balearic Islands and Chafarinas Islands, where tardigrades have been found. Identification number corresponding to localities in Table III. "X" marks new localities where have been found new tardigrade records for the Iberian Peninsula, the Balearic Islands and Chafarinas Islands. Region "A" includes next localities: 120b, 137, 138, 153,159, 169, 170, 172, 173, 177, 183, 184, 196, 206, 207, 213, 216, 222, 237.

Fig. 1.- Localidades muestreadas en la Península Ibérica, Islas Baleares e Islas Chafarinas, donde se han encontrado tardígrados. El número de identificación de las localidades se corresponde con el de la Tabla III. Hemos marcado con una "X" las nuevas localidades de la Península Ibérica, Islas Baleares e Islas Chafarinas donde hemos encontrado tardígrados. La región "A“ incluye las siguientes localidades: 120b, 137, 138, 153,159, 169, 170, 172, 173, 177, 183, 184, 196, 206, 207, 213, 216, $222,237$.

Valencia; Madrid Moreno, 1911) to 1996 (Villora Moreno \& De Zio Grimaldi, 1996), a zoogeographic study of marine tardigrades from the Chafarinas Islands (Mediterranean Sea, off North Africa).

These data are completed with information from new material collected by Dr. A. I. Camacho from groundwater (interstitial environments and caves) from Cantabria, Jaén and Madrid (North, South and Centre of Spain), and from stratified sampling (by the author) of leaf-litter, rock mosses, trunk lichens and mosses, and freshwater algae from different points in the province of Madrid (centre of Spain). In addition to the customary habitats (with respect to Tardigrada phylum) such as leaf-litter, mosses, and lichens from different substratums as: trunks and rocks; we also sampled such unusual habitats as interstitial ones associated with epigean streams or freshwaters algae, and habitats not previously sampled in the Iberian Peninsula (with respect to Tardigrada phylum), like interstitial habitats associated with subterranean rivers or gours in caves. 
Table II. - Iberian Tardigrada species classified by class, order, family, genus and species. This table contains the following information: Tardigrada species names, supraspecific categories, synonymies, number of localities (corresponding to that in Table III and Fig. 1) in the Iberian Peninsula, the Balearic Islands and Chafarinas Islands, habitats-substratums where found and the first published record in the Iberian Peninsula per each species. Marked with a box new records for Iberian Peninsula, the Balearic Islands and Chafarinas Islands (inside brackets, number of specimens found). $\mathrm{M}=$ moss, $\mathrm{L}=$ lichen, $\mathrm{H}=$ leaf-litter, Hep= hepatic, $\mathrm{R}=$ rock, $\mathrm{E}=$ soil, $\mathrm{Ac}=$ freshwater, $\mathrm{Ch}=$ pool, $\mathrm{Es}=$ pond, $\mathrm{TA}=$ water tank, $\mathrm{SM}=$ marine sediment, $\mathrm{AM}=$ marine algae, $\mathrm{Cirr}=$ barnacle, $\mathrm{ISR}=$ interstitial habitats from subterranean rivers, ICR $=$ interstitial habitats from cave rivers. ${ }^{*}=$ habitat-substratum not specified in article.

Tabla II.- Las especies ibéricas del phylum Tardigrada clasificadas por clase, orden, familia, género y especie. Además se incluye la siguiente información: nombre de las especies, categorías supraespecíficas, sinonimias, número de identificación de las localidades (que se corresponde con los de la Tabla III y la Fig. 1) de la Península Ibérica, Islas Baleares e Islas Chafarinas, hábitat-sustrato donde se han encontrado y artículo donde se citó la especie por primera vez para la Península Ibérica. Se han encuadro las especies que son cita por primera vez para la Península Ibérica, Islas Baleares e Islas Chafarinas (entre paréntesis número de specímenes encontrados). $\mathrm{M}=$ musgo, $\mathrm{L}=$ líquen, $\mathrm{H}=$ hojarasca, $\mathrm{Hep}=$ hepática, $\mathrm{R}=$ roca, $\mathrm{E}=$ edáfico, $\mathrm{Ac}=$ dulceacuícola, $\mathrm{Ch}=\mathrm{charca}$, $\mathrm{Es}=$ estanque, $\mathrm{TA}=$ tanque de agua, $\mathrm{SM}=$ sedimento marino, $\mathrm{AM}=$ alga marina, $\mathrm{Cirr}=$ cirrípedo, $\mathrm{ISR}=$ hábitats intersticiales asociados a ríos subterraneos, ICR= hábitats intersticiales de ríos cavernícolas. *= hábitat-sustrato no especifícado en el artículo.

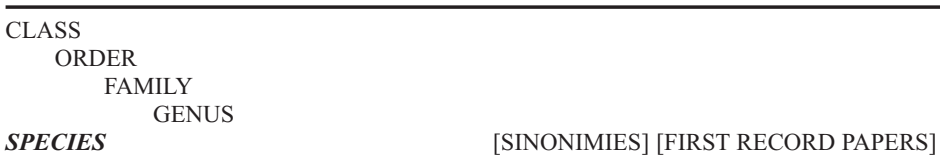

LOCALITIES

HABITAT-SUBSTRATUMS

EUTARDIGRADA Marcus, 1927

APOCHELA Schuster, Nelson, Grigarick \& Christenberry, 1980

Milnesiidae Ramazzotti, 1962 Milnesium Doyére, 1840

Milnesium tardigradum Doyére, 1840 [Heinis, 1908; Da Cunha, 1941]

$11,16,18,20,25,26,27,38,43,50,60,78,85,94,95,97,108,112,114,120,123,124,128,134,137,141,143,144,145$,

$148,151,157,158,159,161,163 \mathrm{~b}, 164,169,172,173,177,179,180,182,193,211,219,225,228,231,234,241,245$

$\mathrm{M}, \mathrm{MR}, \mathrm{L}, \mathrm{LA}, \mathrm{M}+\mathrm{L}, \mathrm{M}+\mathrm{L} \mathrm{A}$

$\mathrm{M}+\mathrm{L} \mathrm{R}, \mathrm{H}$

PARACHELA Schuster, Nelson, Grigarick \& Christenberry, 1980

Calohypsibiidae Pilato, 1969

Calohypsibius Thulin, 192

Calohypsibius ornatus (Richters, 1900) [Hypsibius (Calohypsibius) ornatus (Richters, 1900); Hypsibius (Calohypsibius) ornatus + var. spinosissima Marcus, 1936; Hypsibius (Calohypsibius) ornatus typicus + ornatus carpaticus Bartos, 1940; Hypsibius (Calohypsibius) armatus Bartos; Hypsibius (Calohypsibius) intermedius Mihelcic, 1939; Macrobiotus ornatus var. spinifer Richters, 1900 ] [Da Cunha, 1941] $40,46,49,60,66,112,139,140,150,151$

$\mathrm{L}, \mathrm{M}+\mathrm{L}, \mathrm{M}+\mathrm{L}$ A, F+M+L R

Calohypsibius placophorus (Da Cuhna, 1943) [Hypsibius (Calohypsibius) placophorus Da Cuhna, 1943] [Da Cunha, 1943]

$46 \mathrm{M}+\mathrm{L}, \mathrm{M}+\mathrm{L}$ A

Calohypsibius verrucosus (Richters, 1900) [Hypsibius (Calohypsibius) scabrosus Thulin, 1928; Hypsibius (Calohypsibius) verrucosus Richters, 1900;

$20,39,40,114,151$ Calohypsibius scabrosus Thulin, 1928][Da Cunha, 1947 a]

L, LR, MA

Hypsibiidae Pilato, 1969

Astatumen Pilato, 199

Astatumen trinacriae (Arcidiacono, 1962 [Itaquascon trinacriae Arcidiacono, 1962] [Maucci \& Durante Pasa, 1985]

$39,112,114,115,120 \mathrm{~b}, 137,138,139,140,141$

$\mathrm{M}+\mathrm{L}$

Diphascon Plate, 1889

Adropion Pilato, 1987

Diphascon (Adropion) prosirostre Thulin, 1928 [Maucci \& Durante Pasa, 1984] 29, 139

Diphascon (Adropion) scoticum Murray, 1905 [Hypsibius (Diphascon) scoticus Marcus, 1936; Diphascon crozetense Richters, 1907; Hypsibius scoticus Thulin, 1911; 127,239 Diphascon scoticus Dastych, 1974] [Rodríguez Roda, 1946]

Diphascon Pilato, 1987

Diphascon (Diphascon) alpinum Murray, 1906 [Hypsibius (Diphascon) alpinum Marcus, 1936; Hypsibius (Diphascon) alpinus Murray, 1906] [Da Cunha, 1944 a] $40,44,67,104,114,131,132,156$ M, MA, MR, M+L A, M+L R

Diphascon (Diphascon) chilenense Plate, 1888 [Hysibius (Diphascon) chilenensis Marcus, 1936] [Maucci \& Durante Pasa, 1984] $39,43,44,115,139,141,152,153,154,155$

Diphascon (Diphascon) nobilei (Binda, 1969) ～[Hypsibius (Diphascon) nobilei Binda, 1969][ Maucci \& Durante Pasa, 1984] 104

Diphascon (Diphascon) nodulosum (Ramazzotti, 1957) [Hypsibius (Diphascon) nodolosum Ramazzotti, 1957] 255 (16 specimens found)

H, LT (Pinus sylvestris)

Diphascon (Diphascon) oculatum Murray, 1906 [Diphascon canadensis Murray, 1910; Hypsibius vancouverensis Thulin, 1911; Hypsibius (Diphascon) oculatus

$71,128,136,239$

Murray] [Rodríguez Roda, 1946]

LA, LE, LR, MR 


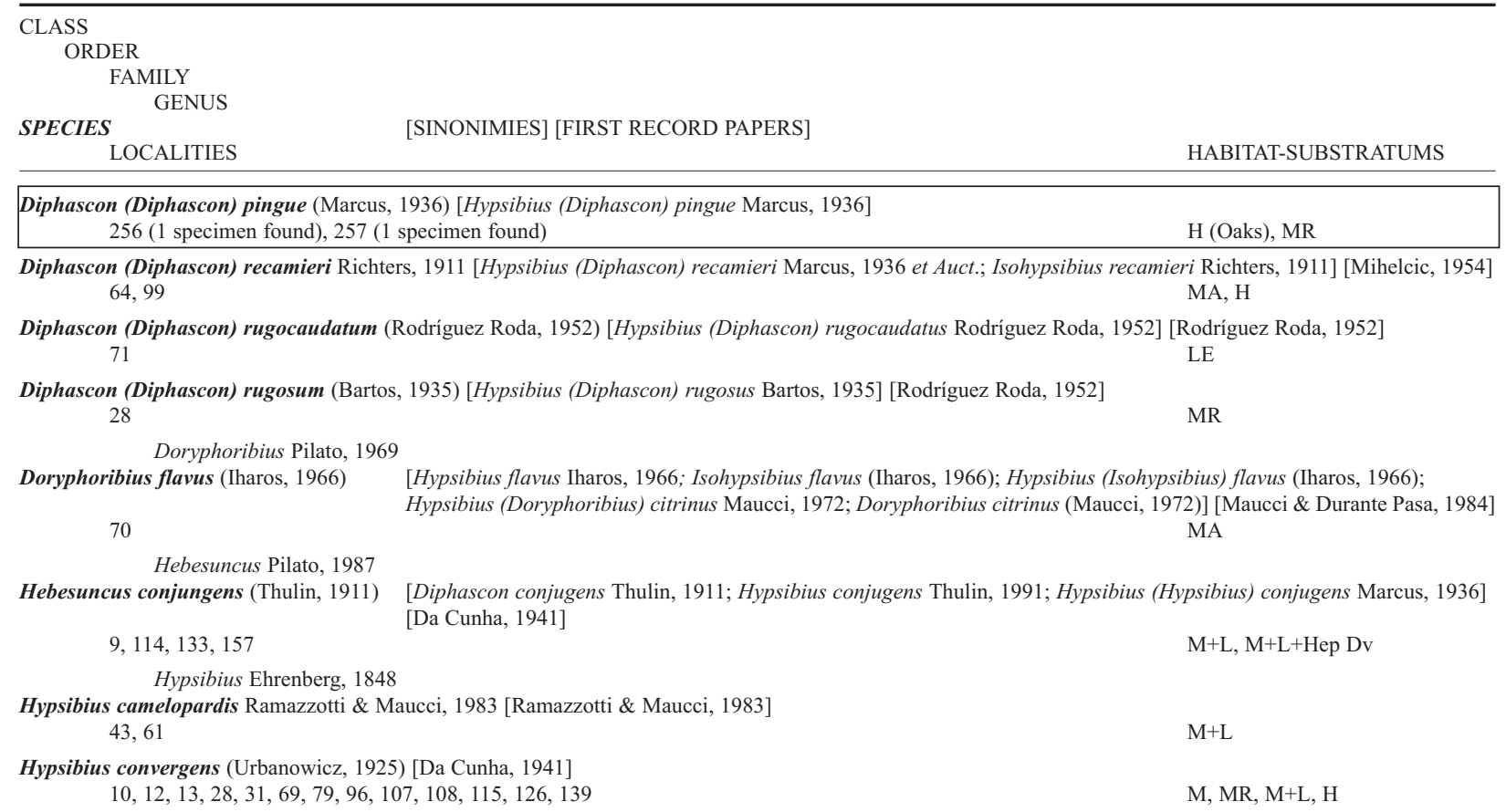

Hypsibius dujardini (Doyère, 1840) [Macrobiotus dujardin Doyére, 1840; Macrobiotus lacustris + palustriz Dujardin, 1851; Macrobiotus tetradactylus Lance, 1896; Macrobiotus murrayi Richters, 1907; Macrobiotus breckneri Richters, 1910; Macrobiotus samoanus Richters, 1908; $45,64,67,84$ Macrobiotus ursellus Della Valle, 1915; Hypsibius dujardini + murrayi Marcus, 1929] [Rodríguez Roda, 1949]

Hypsibius microps Thulin, 1928 $67,74,139,152,175,246$ M, MAc, MR, H

[Hypsibius pallidus Cuénot, 1932 (no Thulin, 1911)] [Maucci \& Durante Pasa, 1984]

Hypsibius pallidus Thulin, 1911 $10,29,31,40,50,66,79,114,126,134,239,242 \quad$ FR, LA, LR, M, MR, M+L,

Hypsibius scabropygus Cuénot, 1929 [Rodríguez Roda, 1952] 71,72

Hypsibius scabrosus (Murray, 1911) 40,66 Isohypsibius Thulin, 1928

Isohypsibius annulatus (Murray, 1911)

$$
\text { 29, 84, } 109
$$

Isohypsibius josephi (Iharos, 1964) 163

Isohypsibius lunulatus (Iharos, 1966) $35,111,175$

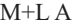
$\mathrm{M}+\mathrm{L} A$

MA, M+L

LA, LE

[Macrobiotus ornatus Richters; Hypsibius verrucosus (Thulin, 1991 and Marcus, 1928); Calohypsibius scabrosus Thulin, 1928] [Da Cunha, 1944 a]

\section{LA, LR, M+L}

[Macrobiotus annulatus Murray, 1905; Hypsibius annulatus Thulin, 1911; Hypsibius (Isohypsibius) annulatus Marcus 1929 y 1936; Hypsibius (Isohypsibius) annulatus Ramazzotti, 1962 y 1972] [Rodríguez Roda, 1949]

[Hypsibius (Isohypsibius) josephi Iharos, 1964] [Maucci \& Durante Pasa, 1985] $\mathrm{Mac}$

M+L

[Hypsibius (Isohypsibius) lunulatus Iharos, 1966] [Maucci \& Durante Pasa, 1984]

$\mathrm{M}+\mathrm{L}$

Isohypsibius mammillosus (Iharos, 1964) [Hypsibius (Isohypsibius) mammillosus Iharos, 1964] [Fontoura, 1982] 107

Isohypsibius marcellinoi (Binda \& Pilato, 1971) [Hypsibius (Isohypsibius) marcellinoi Binda \& Pilato, 1971] 258 (1 specimen found)

Isohypsibius monstruosus Maucci, 1991 [Maucci, 1991]

$$
13
$$
montanus (Mihelcic, 1938) [Hypsibius (Isohypsibius) josephi Iharos, 1964; Hypsibius (Isohypsibius) montanus Mihe

Isohypsibius nodosus (Murray, 1907) ～[Hypsibius (Isohypsibius) nodosus Murray, 1907] [Mihelcic, 1955] 65

Isohypsibius prosostomus (Thulin, 1928) [Hypsibius (Isohypsibius) prosostomus Thulin, 1928] [Da Cunha, 1948] $13,44,51,77,107,126,135,183,184$ Algae from a freshwater pond

Isohypsibius sattleri (Richters, 1902) [Isohypsibius bakonyiensis Iharos, 1964; Hypsibius (Isohypsibius) sattleri (Richters, 1902) et Auct. (partim)] [Da Cunha, 1947a] 29, 65, 73, 118, 221

Itaquascon Barros, 1939

Itaquascon ramazzottii Iharos, 1966 [Fontoura, 1981] 107,114

\section{M}

cic, 1938] [Lewin Osorio, 1984]

MA

$\mathrm{M}, \mathrm{M}+\mathrm{L} \mathrm{A}, \mathrm{M}+\mathrm{L} \mathrm{R}$ 


\begin{tabular}{l} 
CLASS \\
$\begin{array}{l}\text { ORDER } \\
\text { FAMILY } \\
\text { GENUS }\end{array}$ \\
$\begin{array}{l}\text { SPECIES } \\
\text { LOCALITIES }\end{array}$ \\
\hline
\end{tabular}

Pseudobiotus Schuster, Nelson, Grigarick \& Christenberry, 1980

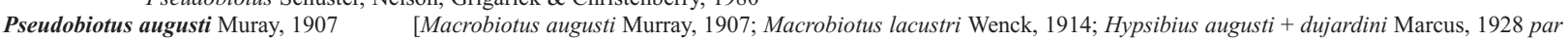
tim; Isohypsibius megalonyx + augusti Thulin, 1928; Hypsibius (Isohypsibius) megalonyx + augusti Marcus, 1936] [Mihelcic, 1954]

23

MR

Ramazzottius Binda \& Pilato, 1986

Ramazzottius novemcinctus Marcus, 1936 [Hypsibius novemcinctus Marcus, 1936] [McInnes, 1991]

MR

Ramazzottius oberhaeuseri (Doyére, 1840) [Hypsibius oberhauseri Doyére, 1840; Macrobiotus oberhaeuser Doyére, 1840; Macrobiotus granulatus Richters, 1908; Macrobiotus spallanzanii Della Valle, 1915] [Da Cunha, 1941]

$10,13,26,31,43,45,48,65,97,101,105,112,114,122,129,130,131,140,141,151,155,157,180,182,211 \quad$ L, LA, LR, MA, M+L, M+L A, $\mathrm{M}+\mathrm{L} R$

Macrobiotidae Thulin, 1928

Dactylobiotus Schuster, Nelson, Grigarick \& Christenberry, 1980

Dactylobiotus ambiguus (Murray, 1907) [Macrobiotus ambiguus Murray, 1907] [Rodríguez Roda, 1952] $80,81,109$

Dactylobiotus dispar (Murray, 1907) ～[Macrobiotus dispar Murray, 1907 et Auct.] [Rodríguez Roda, 1947] $82,83,84,93$

Mes, MTur

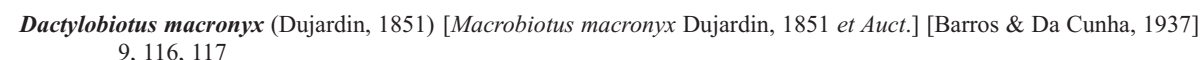
$9,116,117$

Ch, Es, AEs, MAc

Dactylobiotus parthenogeneticus Bertolani, 1981

22 (6 specimens found), 253 ( 1 specimen found) $\quad$ ISR, ICR

$\begin{array}{cc}\text { Dactylobiotus selenicus } \text { Bertolani, } 1981 & \text { ISR } \\ 254 \text { (4 specimens found) }\end{array}$

Macrobiotus Schultze, 1833

Macrobiotus areolatus Murray, 1907 [Macrobiotus echinogenitus Richters, 1903 partim (no M. echinegitus Richters, 1904); Hypsibius areolatus Marcus 1936; Macrobiotus richtersi type 2 Petersen, 1951 (no Murray); Macrobiotus harmsworthi Hallas, 1972 partim (no Murray)] [Barros \& Da Cunha, 1937]

$9,18,19,21,43,78,85,94,107,113,120,146,147,148,158,160,163,164,175,179,196,198,199,200,201,203 \quad \mathrm{M}, \mathrm{M}+\mathrm{L}$

Macrobiotus baltatus McInnes, 1991 [McInnes, 1991] $67,89,90$

Macrobiotus dubius Murray, 1907 [Rodríguez Roda, 1952]
109

Macrobiotus dubius Murray, 1907 [Rodríguez Roda, 1952]
109

MR

Macrobiotus echinogenitus Richters, 1904 [Macrobiotus crenulatus Murray, 1907 (no Macrobiotus echinogenitus Richters, 1903)] [Heinis, 1908; Da Cunha, 1941] $16,46,66,72,86,87,185,239 \quad$ MA, MDulc, MR, M+L A

Macrobiotus furciger Murray, 1906 [Macrobiotus furcatus Murray, 1906 (nec Ehrenberg, 1859); Macrobiotus ehrenbergi Heinis, 1921] [Maucci, 1991] 13

Macrobiotus grandis Richters, $1911 \quad$ [Lewin Osorio, 1984] 95

$\mathrm{M}$

$\mathrm{M}$

Macrobiotus harmsworthi Murray, 1907 [Macrobiotus echinogenitus Richters, 1903 partim; Macrobiotus tetrodon + astronensis Della Valle, 1915; Macrobiotus echinogenitus Cuénot, 1932 (no Richters); Macrobiotus harmsworthi Hallas, 1972 partim] [Rodríguez Roda, 1951] $9,19,21,29,30,43,55,58,61,63,94,96,97,98,99,100,101,103,104,105,114,154,175,180,183,186,187,188,189 \quad \mathrm{E}, \mathrm{H}, \mathrm{M}+\mathrm{L}+\mathrm{Hep} \mathrm{A}, \mathrm{M}+\mathrm{L}+\mathrm{Hep} \mathrm{R}$, $\mathrm{M}+\mathrm{L}, \mathrm{M}+\mathrm{L}$ A, $\mathrm{M}+\mathrm{L} \mathrm{R}$

Macrobiotus hufelandi Schultze, 1833 [Macrobiotus eminens Ehrenberg, 1859; Macrobiotus hufelandii Thulin, 1911; Macrobiotus interruptus Della Valle, 1914; Arctiscon tetradactylum Nitzsch, 1835; Macrobiotus diodon Della Valle, 1915] [Heinis, 1908; Da Cunha, 1941] $8,9,11,16,17,18,19,20,23,25,27,29,30,38,39,40,42,43,44,46,47,50,53,54,62,63,64,65,67,72,78$ $94,96,99,107,108,112,113,114,115,119,120,122,137,138,139,140,141,146,147,148,149,150,151,152$, $153,154,155,157,158,159160,161,162,163,164,165,166,167,168,169,170,171,172,173,174,175,176$, $177,179,180,182,183,184,186,187,188,189,190,191,193,194,196,198,199,200,201,203,205,206,207$, $208,209,210,212,213,214,215,216,217,219,220,221,222,223,224,225,227,228,231,232,233,234,236$, $237,239,241,242,243,244$

Macrobiotus lusitanicus Maucci \& Durante, 1986 [Maucci \& Durante Pasa, 1985] $112,113,114,115$

Macrobiotus montanus Murray, 1910 [Macrobiotus morulatus Bartos, 1936] [Maucci \& Durante Pasa, 1984] 55,100

Macrobiotus occidentalis Murray, 1910 [Barros \& Da Cunha, 1937] $9,11,114$

[Fontoura, 1981] 95, 114

[Macrobiotus aviglianae Robotti, 1970] [Lewin Osorio, 1984]

H, LA, M+L+Hep A, M+L+Hep R, $\mathrm{M}+\mathrm{L}+\mathrm{Hep}$ Dv, MA, ME, MR, $\mathrm{M}+\mathrm{L}, \mathrm{M}+\mathrm{L}$ A

ME, MR, M+L

$\mathrm{LA}, \mathrm{M}+\mathrm{L}$

$\mathrm{L}, \mathrm{M}$ 


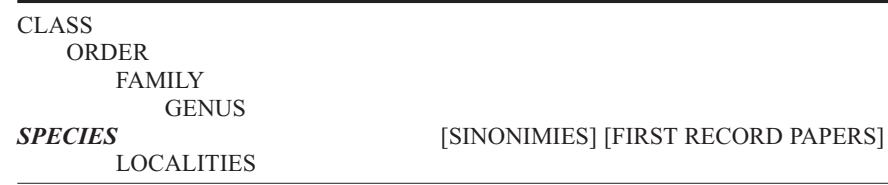

Macrobiotus persimilis Binda \& Pilato, 1972 [Maucci \& Durante Pasa, 1984] $118,119,120,122$

Macrobiotus pseudofurcatus Pilato, 1972 [Maucci \& Durante pasa, 1985]

Macrobiotus recens Cuénot, 1932

[Macrobiotus hufelandi forma recens Marcus 1936;Macrobiotus hufelandi recens Ramazzotti, 1962 and 1972 (no Macrobiotus recens Grigarick, Schuster \& Toftner, 1973; no Macrobiotus recens Horning, Schuster \& Grigarick, 1978)] [Maucci, 1979]

$19,24,25,26,37,40,42,43,52,55,61,114,115,119,120 \mathrm{~b}, 137,139,140,151,155,158,159,161,162,163$ $163 \mathrm{~b}, 171,172,173,177,183,186,190,198,206,211,212,213,214,215,216,217,219,220,221$

$\mathrm{L}, \mathrm{M}, \mathrm{M}+\mathrm{L}, \mathrm{M}+\mathrm{L}$ A, M+L R

Macrobiotus richtersi Murray, 1911 [Macrobiotus harmsworthi Thulin, 1911 (nec Murray); Macrobiotus schultzei Greeff, 1966; Macrobiotus richertsi Marcus, 1936 partim; Macrobiotus richtersii type I Petersen, 1951; Macrobiotus harmsworthi Hallas, 1972 partim (nec Murray)] [Rodríguez Roda, 1946]

$13,18,29,39,42,43,50,62,72,78,83,94,95,97,106,123,124,148,149,160,163,163 \mathrm{~b}, 164,167,172,175$ $179,182,184,186,198,200,201,208,213,221,222,223,224,225,227,228,231,232,233,234,236,241,244$

Macrobiotus tetraplacoides Fontoura, 1981 [Fontoura, 1981]

180

Minibiotus Schuster, Nelson, Grigarick \& Christenberry, 1980

Minibiotus furcatus (Ehrenberg, 1859) [Macrobiotus furcatus Ehrenberg, 1859; Macrobiotus luteus Thulin, 1928] [Da Cunha, 1941] $18,24,27,40,44,61,94,114,139,157,180,211$

Minibiotus hufelandioides (Murray, 1910) [Macrobiotus hufelandioides Murray, 1910] [Mihelcic, 1954] 23,64

Minibiotus intermedius (Plate, 1888) [Macrobiotus intermedius Plate, 1888] [Heinis, 1908; Da Cunha, 1941] $9,16,20,24,25,27,29,42,43,44,45,61,67,76,87,89,94,97,104,110,111,112,113,114,120 \mathrm{~b}, 122,137,138$ $139,147,148,150,157,158,160,163,166,169,171,175,183,186,190,193,199,205,206,207,208,209,210,243$

Murrayon Bertolani \& Pilato, 1988

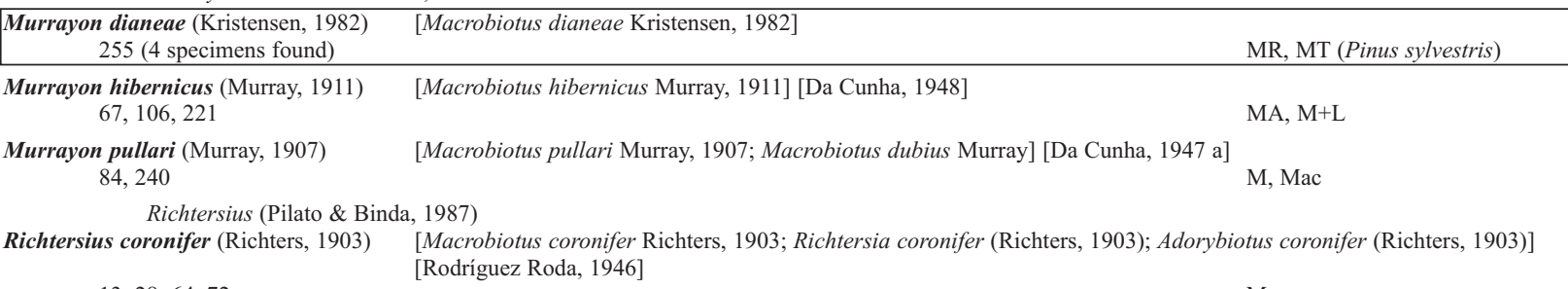
$13,28,64,72$ [Rodríguez Roda, 1946]

LA, LR

LA, M+L, M+L A. M+L R

C, MR

$\mathrm{M}+\mathrm{L}+\mathrm{Hep}, \mathrm{M}, \mathrm{MA}, \mathrm{MR}, \mathrm{M}+\mathrm{L}$, $\mathrm{M}+\mathrm{L}$ R
HETEROTARDIGRADA Marcus, 1927

ARTHROTARDIGRADA Marcus, 1927

Batillipedidae Ramazzzotti, 1962 Batillipes Richters, 1909

Batillipes dicrocercus Pollock, $1970 \quad$ [Maucci \& Durante Pasa, 1984]

SM

Batillipes marcelli Morone de Lucia, R. M., d'Addabbo-Gallo, M. \& Grimaldi de Zio, S., 1988 [Villora Moreno \& De Zio Grimaldi, 1996]

250

[Rodríguez Roda, 1947]

[Villora Moreno, 1993]

Batillipes pennaki Marcus, 1946 249

[Villora Moreno, 1993]

Batillipes phreaticus Renaud-Debyser, 1959 [Villora Moreno, 1993] $247,248,249$

Halechiniscidae Ramazzotti, 1962

Actinarctus Schulz, 1935

Actinarctus doryphorus Schulz, 1935 [Villora Moreno \& De Zio Grimaldi, 1996] 252

Actinarctus physophorus Grimaldi de Zio, S., d'Addabbo Gallo, M., Morone de Lucia, R. M., Vacoarella, R. \& Grimaldi, P., 1982 252

[Villora Moreno \& De Zio Grimaldi, 1996]

SM

Bathyechiniscus Steiner, 1926

Bathyechiniscus tetronyx Steiner, 1926 [Styraconyx sargassi Thulin, 1942] [Rodriguez Roda, 1947] $1,2,3$ 


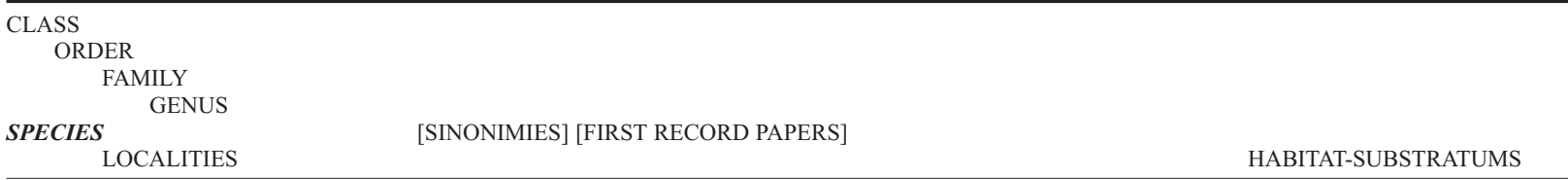

Florarctus Delamare-Deboutteville \&Renaud-Mornant, 1965

Florarctus acer Renaud-Mornant, 1989 [Villora Moreno \& De Zio Grimaldi, 1996] 252

Florarctus asper Renaud-Mornant, 1989 [Villora Moreno \& De Zio Grimaldi, 1996] 251

SM

SM

SM 250

Florarctus hulingsi Renaud-Mornant, 1976 [Villora Moreno \& De Zio Grimaldi, 1996] 250,251

Florarctus stellatus Renaud-Mornant, 1989 [Villora Moreno \& De Zio Grimaldi, 1996]

Halechiniscus Richters, 1908

Halechiniscus chafarinensis Grimaldi \& Villora Moreno, 1996 [De Zio Grimaldi \& Villora Moreno, 1996]

Halechiniscus greveni Renaud-Mornant \& Deroux, 1976 [Villora Moreno \& De Zio Grimaldi, 1996]

[Villora Moreno \& De Zio Grimaldi, 1996]

[Villora Moreno \& De Zio Grimaldi, 1996]

cus remanei Schulz, 195

Styraconyx Thulin, 1942

Styraconyx craticulus (Pollock, 1983) [Bathyechiniscus craticulus Pollock, 1983] [Villora Moreno \& De Zio Grimaldi, 1996]

Stygarctidae Schulz, 195 Stygarctus Schulz, 1951

Stygarctus bradypus Schulz, 1951 [Villora Moreno \& De Zio Grimaldi, 1996] 251

ECHINISCOIDEA Marcus, 1927

Echiniscidae Thulin, 1928

Bryodelphax Thulin, 1928

Bryodelphax mateusi (Fontoura, 1982) [Echiniscus (Bryodelphax) mateusi Fontoura, 1982] [Fontoura, 1982] 107

Bryodelphax parvulus Thulin, 1928 [Echiniscus (Bryodelphax) parvulus Thulin, 1928; Echiniscus intermedius Murray, 1910; Thulin, 1911] [Da Cunha, 1941] $8,9,10,11,28,146,147,148,149$

$\mathrm{M}+\mathrm{L}, \mathrm{M}+\mathrm{L} \mathrm{A}$

Bryodelphax tatrensis (Weglarska, 1959) [Echiniscus (Bryodelphax) tatrensis Weglarska, 1959] [Maucci \& Durante Pasa, 1984]

12,13

Cornechiniscus Maucci \& Ramazzotti, 198

Cornechiniscus lobatus (Ramazzotti, 1943b) [Pseudechiniscus cornutus forma lobata Ramazzotti, 1943b; Pseudechiniscus cornutus lobatus Ramazzotti, 1962 y 1972; Pesudechiniscus cornutus Mihelcic, 1966; Dastych, 1972 (no Richters, 1906)] [Maucci \& Durante Pasa, 1984]

1972; Pesudechiniscus cornutus Mihelcic, 1966; Dastych, 1972 (no Richters, 1906)]

Cornechiniscus subcornutus Maucci \& Ramazzotti, 1981 [Maucci \& Ramazzotti, 1981]

Echiniscus Schultze, 1840

Echiniscus bisculptus Maucci, 1983 [Maucci \& Durante Pasa, 1985] 165

[Maucci \& Durante Pasa, 1984]

$\mathrm{M}+\mathrm{L}$

$\mathrm{M}+\mathrm{L}$

$18,19,158,159$

[Echiniscus ramazzotti Binda \& Pilato, 1969; Echiniscus punctulatus Mihelcic, 1955; Echiniscus bellus Mihelcic, 1955] [Da Cunha, 1941]

$\mathrm{MA}, \mathrm{M}+\mathrm{L}$

$20,65,137$

[Echiniscus punctulatus Mihelcic, 1955; Echiniscus bellus Mihelcic, 1967; Echiniscus (Echiniscus) canadensis Marcus, 1936] [Da Cunha, 1941]

$18,19,21,22,23,24,25,26,27,43,65,94,119,139,158,159,161,162,163,164$

$\mathrm{L}, \mathrm{MR}, \mathrm{M}+\mathrm{L}, \mathrm{M}+\mathrm{L} \mathrm{R}$

Echiniscus granulatus (Doyère, 1840) [Emydium granulatum + granulosum Doyére, 1840; Echiniscus crassus Richters et al., 1904; Echiniscus abanti, Maucci, 1972; Echiniscus fortis Bartos, 1935] [Rodríguez Roda, 1946]

$8,12,13,23,28,29,30,31,33,34,35,107,108,160$

Echiniscus lichenorum Maucci, 1983 [Maucci, 1983]

122,166

Rodríguez Roda, 1949]

23, 25, 37, 38, 139, 158,163b, 167
$\mathrm{M}, \mathrm{ME}, \mathrm{MR}, \mathrm{M}+\mathrm{L}$

LA, LE, M+L

$\mathrm{M}+\mathrm{L}, \mathrm{M}+\mathrm{L}$ R 


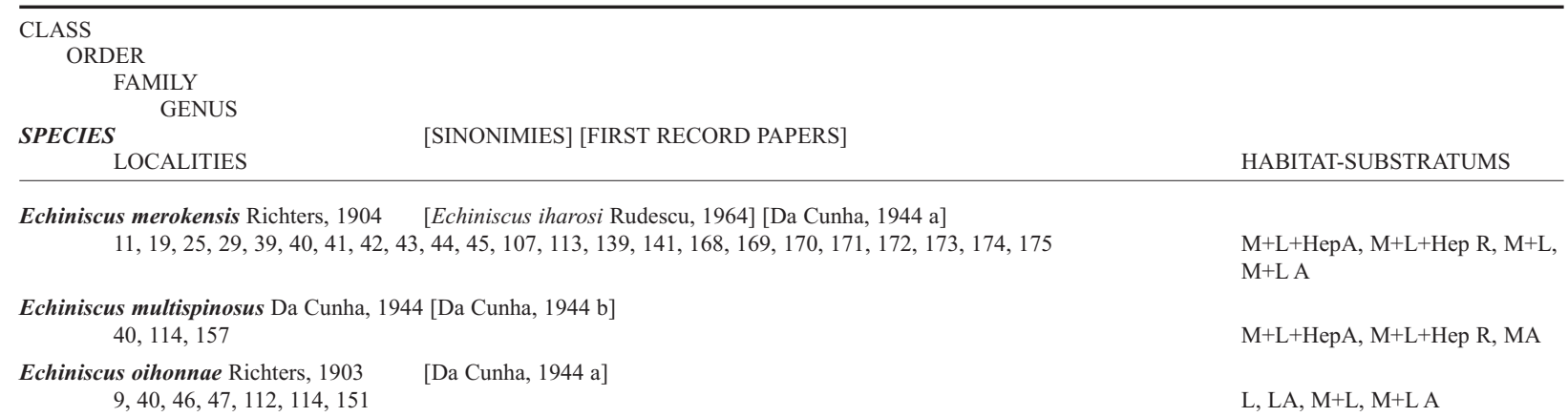

Echiniscus quadrispinosus Richters, 1902 [Echiniscus scrofa Richters, 1902; Echiniscus (Echiniscus) quadrispinosus Marcus, 1936] [Heinis, 1908; Da Cunha, 1941] $17,48,49,50,124,151,175$

$\mathrm{L}, \mathrm{M}, \mathrm{M}+\mathrm{L}$

Echiniscus scabrospinosus Fontoura, 1982 [Fontoura, 1982]

107

Echiniscus sinuloides Murray, 1907 51

Echiniscus spinulosus (Doyère, 1840) $9,35,52,146$

Echiniscus testudo (Doyère, 1840) $14,35,52,53,55,56,58,59$

[Maucci \& Durante Pasa, 1984]

[Maucci \& Durante Pasa, 1984]

$\mathrm{M}+\mathrm{L}$

[Emydium testudo Doyére, 1840; Echiniscus bellermanni Schultze, 1840; Echiniscus inermis Richters, 1902; Echiniscus trifilis Rahm, 1921; Echiniscus filamentosus mongoliense Iharos, 1973 (no E. filamentosus Plate, 1888 )] [Rodríguez Roda, 1949] $\mathrm{MR}, \mathrm{M}+\mathrm{L}, \mathrm{M}+\mathrm{L} \mathrm{R}$

Echiniscus trisetosus Cuénot, 1932 [Echiniscus granulatus Murray, 1905 (no Echiniscus granulatus Doyére); Echiniscus granulatus Murray, 1929] [Rodríguez Roda, 1946]

$8,18,20,21,23,25,26,30,39,45,60,61,62,63,64,66,164,176,177,179$

$\mathrm{H}, \mathrm{L}, \mathrm{M}, \mathrm{MA}, \mathrm{ME}, \mathrm{MR}, \mathrm{M}+\mathrm{L}$, $\mathrm{M}+\mathrm{L} \mathrm{R}$

Hypechiniscus Thulin, 1928

Hypechiniscus gladiator (Murray, 1905) [Echiniscus gladiator Murray, 1905; Echiniscus (Hypsibius) gladiator Marcus, 1929;

39,66 Parechiniscus unispinosus Da Cuhna, 1947b] [Maucci \& Durante Pasa, 1984]

Parechiniscus Cuénor, 192

Parechiniscus chitonides Cuénot, 1926 [Rodríguez Roda, 1946] 8,72

Parechiniscus unispinosus Da Cunha, 1947b [Da Cunha, 1947 b] 39,66

Pseudechiniscus Cuénot, 1926

Pseudechiniscus clavatus Mihelcic, 1955 [Mihelcic, 1955] 65

Pseudechiniscus facettalis (Petersen, 1951) [Pseudechiniscus suillus forma facettalis Petersen, 1951; Pseudechiniscus pseudocoronifer forma facettales Maucci, $29,67,68,69,120 \mathrm{~b}, 237$ 1954; Pseudechiniscus suillus facettalis Ramazzotti, 1962 y 1972] [Maucci \& Durante Pasa, 1984] iniscus insolitus Maucci, 1988 [Maucci, 1991] 13 $\mathrm{MA}, \mathrm{MR}, \mathrm{M}+\mathrm{L}$

Pseudechinisc iniscus pseudocoronifer Ramazzotti, 1943a [Maucci \& Durante Pasa, 1984]

Pseudechiniscus suillus (Ehrenberg, 1853) [Echiniscus suillus Ehrenberg, 1853; Echiniscus mutabilis Murray, 1905; Echiniscus arctomys Auct. (no Ehrenberg) 1902-1910] [Da Cunha, 1941]

$9,11,12,20,25,31,40,46,47,51,66,71,72,73,74,75,76,77,78,79,107,112,113,114,139,140,141,148$, $150,151,152,153,158,162,164,180,217$

$\mathrm{L}, \mathrm{LA}, \mathrm{M}, \mathrm{MR}, \mathrm{M}+\mathrm{L}, \mathrm{M}+\mathrm{L} \mathrm{A}$, $\mathrm{M}+\mathrm{L} R$

$\mathrm{M}$

us spinuloides (Murray, 1907) [Echiniscus spinuloides Murray, 1907] [Maucci \& Durante Pasa, 1984]

Echiniscoididae Kristensen \& Hallas, 1980 Echiniscoides Plate, 1889

Echiniscoides sigismundi hispaniensis Kristensen \& Hallas, 1980 [Kristensen \& Hallas, 1980] $6,250,25$

Echiniscoides sigismundi mediterraneus Kristensen \& Hallas, 1980 [Kristensen \& Hallas, 1980]
CirrM, SM

AM, LM 
Table III.- Localities list from the Iberian Peninsula, the Balearic Islands and Chafarinas Islands where tardigrade species have been found, with the following information: Country, Autonomic Community (for Spain) or Region (for Portugal), Province (for Spain) or District (for Portugal), identification number for each locality (\#Loc) which corresponds to that on Fig. 1, number of registers (\# registers), number of species (\# spp), types of habitat-substratum sampled for each locality (H-S types) and UTM coordinates for each locality, as precise as possible. $* 1=$ found in literature but not geographic information; $* 2=$ geographic information found in literature but no UTM information. New $=$ new localities sampled with respect to Tardigrada phylum. Other abbreviations as in Table II.

Tabla III.- Lista de las localidades de la Península Ibérica, Islas Baleares e Islas Chafarinas donde se ha encontrado alguna especie del phylum Tardigrada. Presentamos además la siguiente información: país, comunidad autónoma (para España) o región (para Portugal), provincia (para España) o distrito (para Portugal), número de identificación para cada localidad (\#Loc) que se corresponde con los números de la Figura 1, número de registros (\# registers), número de especies (\# spp), tipos de hábitat-sustratos muestreados en cada localidad (H-S types) y coordenadas UTM para cada localidad, tan preciso como ha sido posible. $* 1=$ encontrado en la bibliografía pero sin información geográfica; $* 2=$ encontrada información geográfica en la bibliografía pero sin coordenadas UTM. New= nueva localidad muestreada respecto al phylum Tardigrada. Otras abreviaturas como en la Tabla II.

\begin{tabular}{|c|c|c|c|c|c|c|}
\hline \multicolumn{7}{|c|}{$\begin{array}{l}\text { Country } \\
\text { Autonomic Comunity (SPAin) / Region (Portugal) } \\
\quad \text { Province (SPAin) / District (Portugal) }\end{array}$} \\
\hline ANDORRA & 29 & Andorra & 13 & 11 & $\mathrm{M}$ & $31 \mathrm{TCH}$ \\
\hline $\begin{array}{l}\text { ESPAÑa } \\
\text { Andalucía }\end{array}$ & & & & & & \\
\hline $\begin{array}{l}\text { Granada } \\
\text { Huelva } \\
\text { Jaén } \\
\text { Málaga } \\
\text { Sevilla }\end{array}$ & $\begin{array}{r}69 \\
35 \\
118 \\
61 \\
22 \\
68 \\
70 \\
52 \\
105\end{array}$ & $\begin{array}{l}\text { Venta de Roja*1 } \\
\text { El Zegrí } \\
\text { Loja } \\
\text { Santa Olalla } \\
\text { Despeñaperros } \\
\text { Santa Elena } \\
\text { Gaucín } \\
\text { Atajate } \\
\text { El Ronquillo }\end{array}$ & $\begin{array}{l}2 \\
4 \\
2 \\
6 \\
1 \\
1 \\
3 \\
2 \\
2\end{array}$ & $\begin{array}{l}2 \\
4 \\
2 \\
6 \\
1 \\
1 \\
3 \\
2 \\
2\end{array}$ & MA & $\begin{array}{l}\text { 30SVG4542 } \\
\text { 30SUG9714 } \\
\text { 29SQA24 } \\
\text { 30SVH55 } \\
\text { 30SVH5243 } \\
\text { 30STF95 } \\
\text { 30STF9957 } \\
\text { 29SQB47 }\end{array}$ \\
\hline $\begin{array}{l}\text { Aragón } \\
\text { Huesca }\end{array}$ & $\begin{array}{r}31 \\
59 \\
51 \\
246 \\
111 \\
15 \\
48 \\
74 \\
12 \\
14 \\
75 \\
71 \\
67 \\
101\end{array}$ & $\begin{array}{l}\text { Biescas (between Sarvisé and Fiscal) } \\
\text { Binéfar } \\
\text { Broto } \\
\text { Road between Sarvisé and Fiscal } \\
\text { Fiscal } \\
\text { Huesca } \\
\text { Jaca } \\
\text { Linas de Broto } \\
\text { Paso Foradada } \\
\text { Peraltilla } \\
\text { Sarvisé } \\
\text { Selva de Oza } \\
\text { Valle de Ordesa } \\
\text { El Frasno }\end{array}$ & $\begin{array}{r}5 \\
1 \\
5 \\
1 \\
2 \\
1 \\
2 \\
2 \\
3 \\
2 \\
1 \\
4 \\
10 \\
2\end{array}$ & $\begin{array}{l}5 \\
1 \\
5 \\
1 \\
2 \\
1 \\
2 \\
2 \\
3 \\
2 \\
1 \\
4 \\
9 \\
2\end{array}$ & $\begin{array}{l}\text { M, MR } \\
\text { ME } \\
\text { M+L, L } \\
\text { ME } \\
\text { LE, M+L A } \\
\text { M, MA, MR }\end{array}$ & $\begin{array}{l}\text { 30TYN1923 } \\
\text { 31TBG7537 } \\
\text { 30TYN32 } \\
\text { 30TYN31 } \\
\text { 30TYN3609 } \\
\text { 30TYM1468 } \\
\text { 30TYN01 } \\
\text { 30TYN32 } \\
\text { 31TBG8268 } \\
\text { 30TYM4660 } \\
\text { 30TYN3618 } \\
\text { 30TXN84 } \\
\text { 30TYN32 } \\
\text { 30TXL2586 }\end{array}$ \\
\hline $\begin{array}{l}\text { Cantabria } \\
\text { Cantabria }\end{array}$ & $\begin{array}{r}77 \\
65 \\
253\end{array}$ & $\begin{array}{l}\text { Peña Vieja } \\
\text { Santander } \\
\text { Sumidero del Calderón } \\
\text { (Venta Fresnedo, Sierra de la Collada) }\end{array}$ & $\begin{array}{r}3 \\
8 \\
\text { New }\end{array}$ & $\begin{array}{r}3 \\
7 \\
\text { New }\end{array}$ & $\begin{array}{l}\text { MA, MR } \\
\text { ICR }\end{array}$ & $\begin{array}{l}\text { 30TUN61 } \\
\text { 30TVP31 } \\
\text { 30T0378440-4792244 }\end{array}$ \\
\hline $\begin{array}{l}\text { Castilla La Manch } \\
\text { Ciudad Real }\end{array}$ & $\begin{array}{r}129 \\
145 \\
21 \\
144 \\
53\end{array}$ & $\begin{array}{l}\text { Ciudad Real } \\
\text { Alcázar de San Juan } \\
\text { Almuradiel } \\
\text { Pedro Muñoz } \\
\text { Close to Toledo train station }\end{array}$ & $\begin{array}{l}1 \\
1 \\
3 \\
1 \\
2\end{array}$ & $\begin{array}{l}1 \\
1 \\
3 \\
1 \\
2\end{array}$ & $\begin{array}{l}\mathrm{M}+\mathrm{L} \\
\mathrm{MR} \\
\mathrm{M} \\
\mathrm{LA} \\
\mathrm{MR}\end{array}$ & $\begin{array}{l}\text { 30SVJ1915 } \\
\text { 30SVJ8260 } \\
\text { 30SVH5663 } \\
\text { 30SWJ0461 } \\
\text { 30SVK1212 }\end{array}$ \\
\hline $\begin{array}{l}\text { Castilla León } \\
\text { Ávila } \\
\text { Salamanca }\end{array}$ & $\begin{array}{r}63 \\
30 \\
45 \\
131 \\
104 \\
34 \\
33\end{array}$ & $\begin{array}{l}\text { Arévalo } \\
\text { Ávila } \\
\text { Fuentes de Oñoro } \\
\text { La Orbada } \\
\text { Sancti Spiritus } \\
\text { Aldehuela de la Bóveda } \\
\text { San Román }\end{array}$ & $\begin{array}{l}3 \\
9 \\
5 \\
2 \\
5 \\
1 \\
1\end{array}$ & $\begin{array}{l}3 \\
4 \\
5 \\
2 \\
4 \\
1 \\
1\end{array}$ & $\begin{array}{l}\text { H, ME } \\
\text { H, MA, ME, MR } \\
\text { MR } \\
\text { MA }\end{array}$ & $\begin{array}{l}\text { 30TUL5547 } \\
\text { 30TUL5602 } \\
\text { 29TPE8596 } \\
\text { 30TTL9153 } \\
\text { 29TQF2002 } \\
\text { 29TQF42 } \\
\text { 29TQF53 }\end{array}$ \\
\hline
\end{tabular}




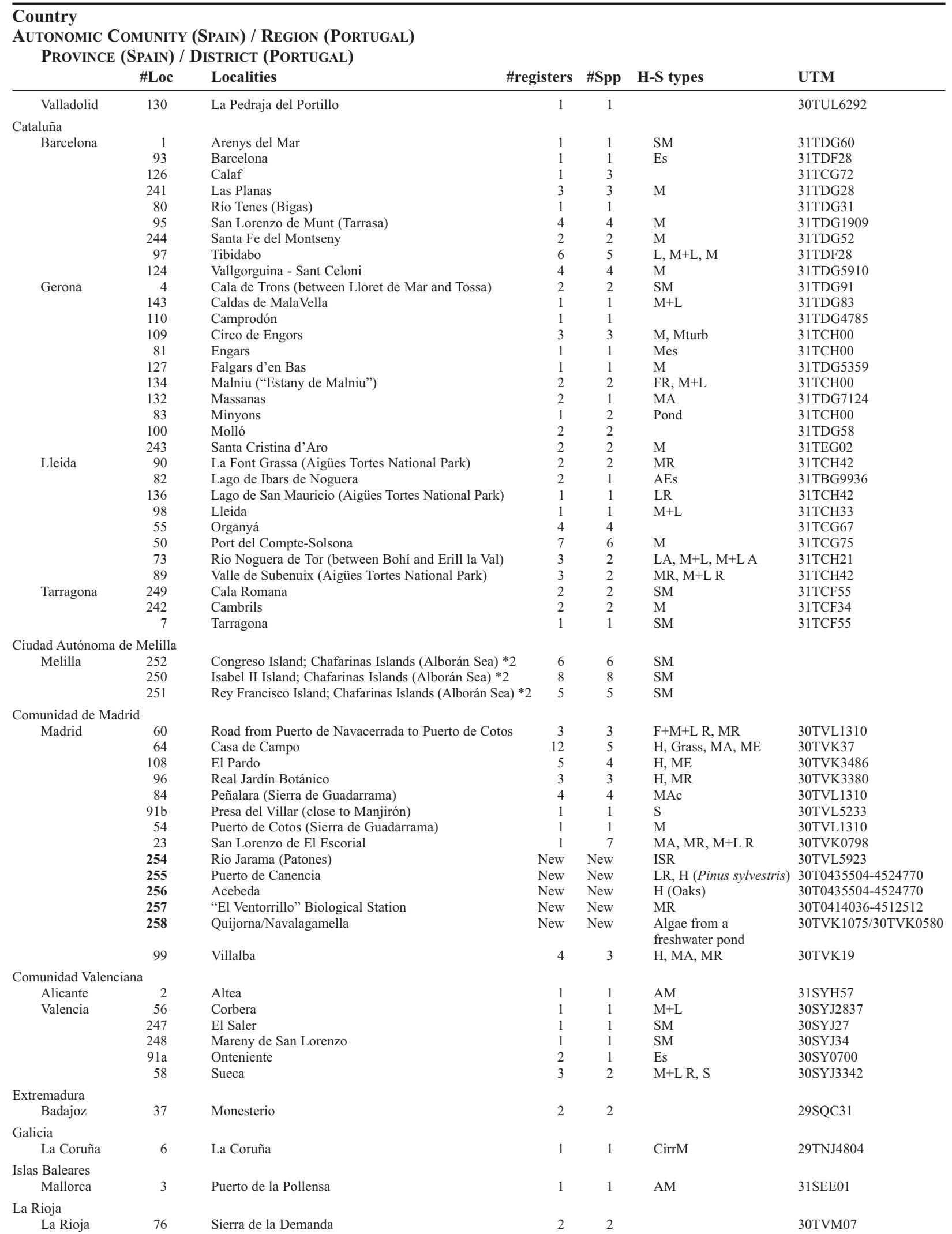




\begin{tabular}{|c|c|c|c|c|c|c|}
\hline \multicolumn{7}{|c|}{$\begin{array}{l}\text { Country } \\
\text { Autonomic Comunity (Spain) / Region (Portugal) } \\
\quad \text { Province (SPain) / District (Portugal) }\end{array}$} \\
\hline \multicolumn{5}{|l|}{ Navarra } & H-S types & UTM \\
\hline \multirow[t]{6}{*}{ Navarra } & 239 & Igaratza mountain refuge (Sierra de Aralar) & 5 & 3 & MR & $30 \mathrm{TWN} 85$ \\
\hline & 185 & Cueva Aparein (entrance; Sierra de Aralar) & 1 & 1 & MR & $30 \mathrm{TWN} 85$ \\
\hline & 191 & Cueva de Basolo (entrance; Sierra de Aralar) & 1 & 1 & MA & $30 \mathrm{TWN} 85$ \\
\hline & 72 & Cueva Putxerri (entrance; Sierra de Aralar) & 9 & 4 & LA, MR & $30 \mathrm{TWN} 85$ \\
\hline & 8 & Between Igaratza refuge and río Unaga (Sierra de Aralar) & 5 & 4 & $\mathrm{M}, \mathrm{MR}$ & $30 \mathrm{TWN} 85$ \\
\hline & 156 & Monte Kilixketa (Sierra de Aralar) & 1 & 1 & M & $30 \mathrm{TWN} 85$ \\
\hline \multicolumn{7}{|c|}{ Navarra/País Vasco } \\
\hline & 28 & Sierra de Aralar & 5 & 4 & $\mathrm{M}, \mathrm{MR}, \mathrm{M}+\mathrm{L}$ & $30 \mathrm{TWN} 85$ \\
\hline \multicolumn{7}{|c|}{ Principado de Asturias } \\
\hline Asturias & 13 & Peña Santa (close to Vegarredonda mountain refuge) & 11 & 10 & M & 30TUN61 \\
\hline \multicolumn{7}{|l|}{$\begin{array}{l}\text { Portugal } \\
\text { Algarye }\end{array}$} \\
\hline \multirow{5}{*}{ Faro } & 122 & Almansil & 6 & 5 & $\mathrm{LA}, \mathrm{M}+\mathrm{L}$ & 29SNB91 \\
\hline & 228 & Besanfim & 3 & 3 & $\mathrm{M}+\mathrm{L}$ & 29SNB21 \\
\hline & 166 & Maria Vinagre & 4 & 3 & $\mathrm{LE}, \mathrm{M}+\mathrm{L}$ & 29SNB24 \\
\hline & 94 & Monchique & 16 & 8 & $\begin{array}{l}\mathrm{L}, \mathrm{LA}, \mathrm{M}+\mathrm{L}+\mathrm{Hep}, \\
\mathrm{M}+\mathrm{L}, \mathrm{M}+\mathrm{L} \mathrm{A}, \mathrm{M}+\mathrm{L} \text { R }\end{array}$ & 29SNB33 \\
\hline & 120 & Vila Real de Santo Antonio & 8 & 4 & $\mathrm{M}+\mathrm{L}$ & 29SPB42 \\
\hline Alto Alentejo & 167 & Conv. de Barra*1 & 4 & 3 & $\mathrm{M}+\mathrm{L}$ & \\
\hline \multirow[t]{11}{*}{ Evora } & 200 & Brotas & 4 & 3 & $\mathrm{M}+\mathrm{L}$ & 29SND70 \\
\hline & 210 & Ciborro & 2 & 2 & $\mathrm{M}+\mathrm{L}$ & 29SNC70 \\
\hline & 179 & Extremos & 6 & 5 & $\mathrm{M}+\mathrm{L}$ & 29SPD20 \\
\hline & 163 & Evora & 7 & 7 & $\mathrm{M}+\mathrm{L}$ & 29SNC96 \\
\hline & $163 b$ & Evora Monte & 4 & 4 & $\mathrm{M}+\mathrm{L}$ & 29SNC96 \\
\hline & 232 & Lavre & 2 & 2 & $\mathrm{M}+\mathrm{L}$ & 29SNC59 \\
\hline & 148 & Montemor & 7 & 7 & $\mathrm{M}+\mathrm{L}$ & 29SNC67 \\
\hline & 186 & Portel & 5 & 5 & $\mathrm{M}+\mathrm{L}$ & 29 SPC14 \\
\hline & 158 & Redondo & 9 & 9 & $\mathrm{M}+\mathrm{L}$ & 29SPC 27 \\
\hline & 162 & S. Manços & 3 & 3 & $\mathrm{M}+\mathrm{L}$ & 29SPC16 \\
\hline & 220 & Vimieiro & 2 & 2 & $\mathrm{M}+\mathrm{L}$ & 29SNC09 \\
\hline \multirow[t]{12}{*}{ Portoalegre } & 119 & Alpalhao & 4 & 4 & $\mathrm{M}+\mathrm{L}$ & 29SPD16 \\
\hline & 161 & Arez & 4 & 4 & $\mathrm{M}+\mathrm{L}$ & 29SPD06 \\
\hline & 62 & Arronches & 4 & 3 & $\mathrm{M}, \mathrm{M}+\mathrm{L}$ & 29SPD43 \\
\hline & 201 & Crato & 3 & 3 & $\mathrm{M}+\mathrm{L}$ & 29SPD14 \\
\hline & 123 & Elvas & 2 & 2 & $\mathrm{M}+\mathrm{L}$ & 29SPD50 \\
\hline & 231 & Fronteira & 3 & 3 & $\mathrm{M}+\mathrm{L}$ & 29SPD12 \\
\hline & 164 & Monforte & 7 & 6 & $\mathrm{M}+\mathrm{L}$ & 29SPD32 \\
\hline & 227 & Montargil & 2 & 2 & $\mathrm{M}+\mathrm{L}$ & 29SND62 \\
\hline & 43 & Niza & 20 & 12 & $\begin{array}{l}\mathrm{LA}, \mathrm{LR}, \mathrm{M}+\mathrm{L}+\mathrm{Hep}, \\
\mathrm{M}, \mathrm{M}+\mathrm{L}, \mathrm{M}+\mathrm{L} \mathrm{A}, \mathrm{M}+\mathrm{L} \mathrm{R}\end{array}$ & 29SPD17 \\
\hline & 42 & Portalegre & 7 & 5 & $\mathrm{M}, \mathrm{M}+\mathrm{L}$ & 29SPD35 \\
\hline & 24 & San Vicente (close to Elvas village) & 5 & 4 & $\mathrm{M}$ & 29SPD50 \\
\hline & 221 & Vale do Peso & 5 & 5 & $\mathrm{MA}, \mathrm{M}+\mathrm{L}$ & 29SPD26 \\
\hline Baixo Alentejo & & & & & & \\
\hline Beja & 203 & Almódavar & 2 & 2 & $\mathrm{M}+\mathrm{L}$ & 29SNB85 \\
\hline & 149 & Alvito & 4 & 3 & $\mathrm{M}+\mathrm{L}$ & 29SNC84 \\
\hline & 233 & Ferreira do Alentejo & 2 & 2 & $\mathrm{M}+\mathrm{L}$ & 29SNC81 \\
\hline & 187 & Mértola & 2 & 2 & $\mathrm{M}+\mathrm{L}$ & 29SPB27 \\
\hline & 175 & Odemira & 9 & 9 & $\mathrm{M}+\mathrm{L}$ & 29SNB36 \\
\hline & 236 & Vila Alva & 2 & 2 & $\mathrm{M}+\mathrm{L}$ & 29SNC93 \\
\hline & 234 & Vila Ruiva & 3 & 3 & $\mathrm{M}+\mathrm{L}$ & 29SNC93 \\
\hline Beira Alta & & & & & & \\
\hline Guarda & 26 & Arrifana & 7 & 5 & $\mathrm{M}, \mathrm{M}+\mathrm{L}$ & 29TPE18 \\
\hline & 198 & Celorico & 4 & 4 & $\mathrm{M}+\mathrm{L}$ & 29TPE39 \\
\hline & 25 & Guarda & 17 & 10 & $\mathrm{M}, \mathrm{M}+\mathrm{L}, \mathrm{M}+\mathrm{L} \mathrm{A}, \mathrm{M}+\mathrm{L} \mathrm{R}$ & 29TPE48 \\
\hline & 44 & Pinzio & 8 & 7 & $\mathrm{M}+\mathrm{L}$ & 29TPE60 \\
\hline & 27 & S. Giao & 9 & 5 & $\begin{array}{l}\text { LA, M+Lhep, M+L, } \\
\text { M+L A, M+L R }\end{array}$ & 29TPE16 \\
\hline & 47 & Vilar Formoso & 3 & 3 & $\mathrm{M}+\mathrm{L}$ & 29TРЕ89 \\
\hline Viseu & 155 & Castro Daire & 4 & 4 & $\mathrm{M}+\mathrm{L}$ & 29TNF82 \\
\hline & 217 & Fail & 3 & 3 & $\mathrm{M}+\mathrm{L}$ & 29TNE89 \\
\hline & 208 & Fornos de Algodres & 3 & 3 & $\mathrm{M}+\mathrm{L}$ & 29SPE20 \\
\hline & 209 & Lordosa & 2 & 2 & $\mathrm{M}+\mathrm{L}$ & 29SNF92 \\
\hline & 141 & Santa Comba Dao & 7 & 7 & $\mathrm{M}+\mathrm{L}$ & 29TNE77 \\
\hline & 139 & Serra do Caramulo & 14 & 14 & $\mathrm{M}+\mathrm{L}$ & 29TNE79 \\
\hline & 174 & Tondela & 2 & 8 & $\mathrm{M}+\mathrm{L}$ & 29TNE78 \\
\hline
\end{tabular}




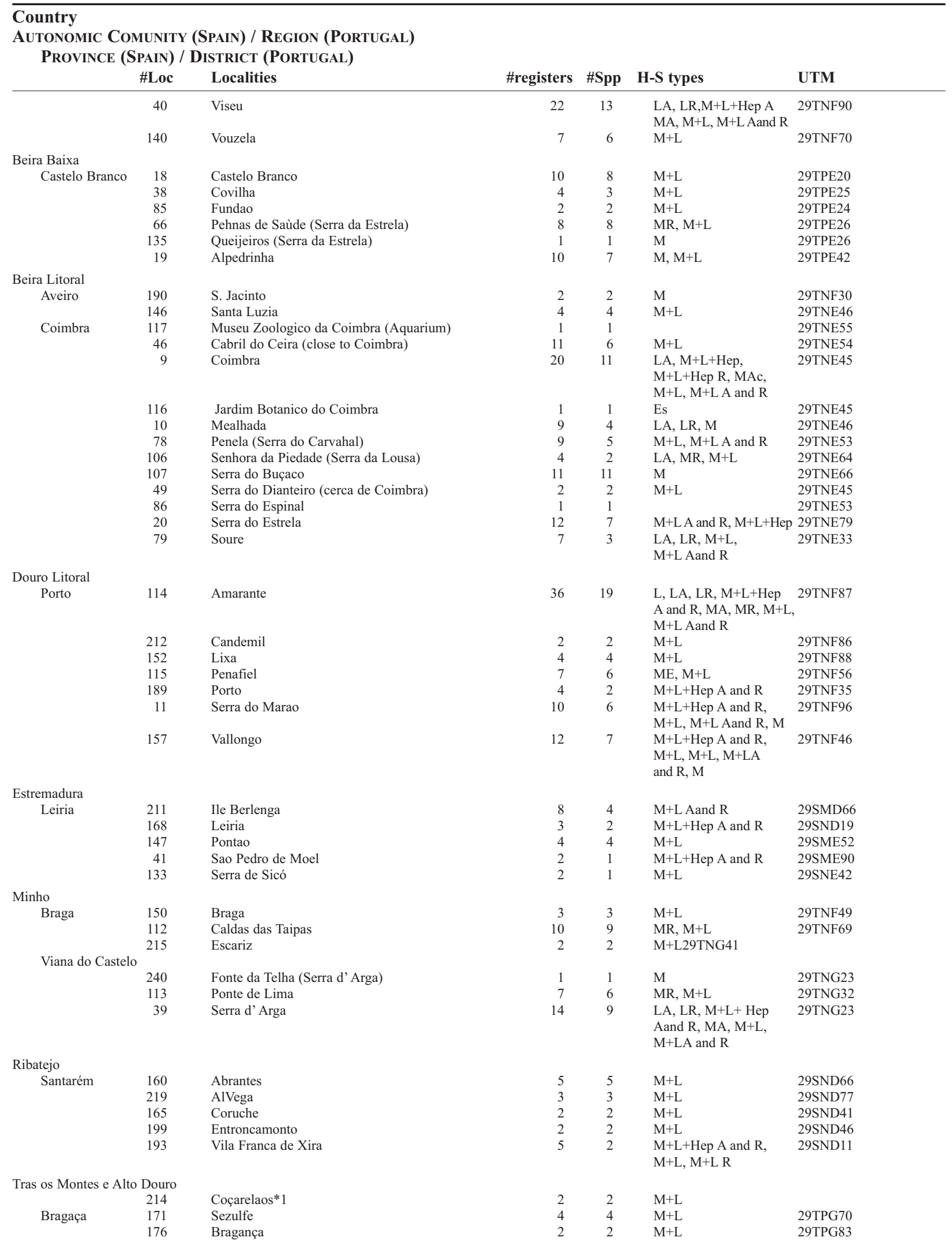




\begin{tabular}{|c|c|c|c|c|c|c|}
\hline \multicolumn{7}{|c|}{$\begin{array}{l}\text { Country } \\
\text { Autonomic Comunity (SPAIn) / Region (Portugal) } \\
\text { Province (SPAin) / District (Portugal) }\end{array}$} \\
\hline & \#Loc & Localities & \#registers & \#Spp & H-S types & UTM \\
\hline & 223 & Milhao & 2 & 2 & $\mathrm{M}+\mathrm{L}$ & 29TPG03 \\
\hline & 205 & Miranda do Douro & 2 & 2 & $\mathrm{M}+\mathrm{L}$ & 29TQF29 \\
\hline & 222 & Mirandela & 2 & 2 & $\mathrm{M}+\mathrm{L}$ & 29TPF59 \\
\hline & 224 & Outeiro & 2 & 2 & $\mathrm{M}+\mathrm{L}$ & 29TQG01 \\
\hline & 213 & Passos & 3 & 3 & $\mathrm{M}+\mathrm{L}$ & 29TPF59 \\
\hline & 225 & Vinhais & 2 & 3 & $\mathrm{M}+\mathrm{L}$ & 29TPG63 \\
\hline \multirow[t]{23}{*}{ Vila Real } & 207 & Águas Frías & 2 & 2 & $\mathrm{M}+\mathrm{L}$ & 29TPG33 \\
\hline & 151 & Albergaria a Velha (Serra do Gerês) & 10 & 9 & $\mathrm{~L}, \mathrm{M}$ & 29SNE93 \\
\hline & 188 & Alijó & 4 & 2 & $\mathrm{M}+\mathrm{L}+\mathrm{Hep} \mathrm{A}$ and $\mathrm{R}$ & 29TPF37 \\
\hline & 138 & Ansiaes & 3 & 3 & $\mathrm{M}+\mathrm{L}$ & 29TPF30 \\
\hline & 196 & Balsa & 2 & 2 & $\mathrm{M}+\mathrm{L}$ & 29TPF39 \\
\hline & 153 & Boticas & 4 & 3 & $\mathrm{M}+\mathrm{L}$ & 29TPG11 \\
\hline & 169 & Bouça & 5 & 4 & $\mathrm{M}+\mathrm{L}$ & 29TPG41 \\
\hline & 206 & Chaves & 3 & 3 & $\mathrm{M}+\mathrm{L}$ & 29TPG22 \\
\hline & 194 & Gerês & 2 & 1 & $\mathrm{M}+\mathrm{L}+\mathrm{Hep} \mathrm{A}$ and $\mathrm{R}$ & 29SNE93 \\
\hline & 184 & Grandais & 3 & 3 & $\mathrm{M}+\mathrm{L}$ & 29TPG40 \\
\hline & 237 & Justes & 2 & 2 & $\mathrm{M}+\mathrm{L}$ & 29TPF28 \\
\hline & 154 & Lamego & 3 & 3 & $\mathrm{M}+\mathrm{L}$ & 29TNF95 \\
\hline & 180 & Mondim de Basto & 14 & 7 & $\begin{array}{l}\mathrm{LA}, \mathrm{LR}, \mathrm{M}+\mathrm{L}+\mathrm{Hep} \\
\mathrm{A} \text { and } \mathrm{R}, \mathrm{M}+\mathrm{L} \mathrm{A} \text { and } \mathrm{R}\end{array}$ & 29TNF88 \\
\hline & 170 & Murça & 2 & 2 & $\mathrm{M}+\mathrm{L}$ & 29TPF28 \\
\hline & 173 & Parada de Cuños & 4 & 4 & $\mathrm{M}+\mathrm{L}$ & 29TNF07 \\
\hline & 177 & Rebordelo & 4 & 4 & $\mathrm{M}+\mathrm{L}$ & 29TPG52 \\
\hline & 216 & Sabroso de Aguiar & 2 & 2 & $\mathrm{M}+\mathrm{L}$ & 29TPF10 \\
\hline & 137 & Sapiaes & 6 & 6 & $\mathrm{M}+\mathrm{L}$ & 29TPG42 \\
\hline & 182 & Sendim & 4 & 4 & $\mathrm{M}+\mathrm{L}$ & 29TPG54 \\
\hline & 183 & Vidago & 5 & 5 & $\mathrm{M}+\mathrm{L}$ & 29TPG11 \\
\hline & 172 & Vila Pouca de Aguiar & 5 & 5 & $\mathrm{M}+\mathrm{L}$ & 29TPF19 \\
\hline & $120 \mathrm{~b}$ & Vila Real & 4 & 4 & $\mathrm{M}+\mathrm{L}$ & 29TPF07 \\
\hline & 159 & Vilarinho de Samarda & 5 & 5 & $\mathrm{M}+\mathrm{L}$ & 29TPF18 \\
\hline \multicolumn{7}{|c|}{ UNITED KINGDOM } \\
\hline & 245 & Gibraltar & 1 & 1 & & $30 \mathrm{STF} 80$ \\
\hline
\end{tabular}

Compiled data were analyzed with two nonparametric analyses, the Spearman correlation and Kruska-Wallis analysis, because data are not normally distributed, in order to locate the highest values of diversity from the studied area and to identify where we should concentrate sampling in future. The analysis were carried out with the STATISTICA 6.0 computer program.

\section{Results}

There are 737 works from all over the World on tardigrades, based on information from Zoological Record. Eighteen scientists elaborated 34 works on the Iberian Peninsula, Balearic Islands and Chafarinas Islands, over a period of 85 years. These articles have described one new genus to science, 10 species and 2 subspecies; only 6 of these works deal with marine tardigrades (Table I and II).

Table II presents tardigrade species found in the Iberian Peninsula, the Balearic Islands and Chafarinas Islands, ordered by class, order, family, genus and species. We have included information about synonymies found within these species, an identification number of localities where each species was found (Table III, Fig. 1), habitat-substratums sampled, article references, and where each species was cited for first time in the Iberian Peninsula.

In the bibliography dealing with the studied area, I found 112 species (in Table II appear 114 taxa but Echiniscoides sigismundi hispaniensis and Echiniscoides sigismundi mediterraneus are two subspecies, and Macrobiotus dubius is asigned as doubtfully species in Ramazzotti, 1972 page 548 and not appear in McInnes, 1994; so we have not taken into account in statistical analysis), 92 limnoterrestrial and 20 marine; $63.4 \%$ of all peninsular species have been found in Portugal, 78.5\% in Spain, 11.8\% in Andorra and $1.1 \%$ in Gibraltar (United Kingdom); 23 peninsular species occur only in Portugal, and 42 only in Spain (plus 14 Spanish species found only in Chafarinas Islands; Table II). There are no published works about marine tardigrades from Portugal. There are genera found only in Spain 
(Doryphoribius, Pseudobiotus, Astatumen, Richtersius, Cornechiniscus and Testechiniscus) or found only in Portugal (Hebesuncus, Itaquascon and Hypechiniscus).

Table III lists localities of the studied area where tardigrades have been found, according to Country, Region (in Portugal) or Autonomic Community (in Spain) and District (in Portugal) or Province (in Spain). I have included identification locality numbers (which correspond to those on Fig. 1), UTM coordinates (when available and as precise as it was possible), number of registers (a register correspond to a record that differs from any other in at least one information field: taxonomical, geographical or/and autecological; it can offer a clue about sampling effort), number of species per locality and type of habitat-substratums studied in each locality where tardigrades occur.

Tardigrades have been found in 228 localities in the studied area (Table III); one is in Andorra, another is in Gibraltar (U. K.), 126 in Portugal and 100 in Spain. On Figure 1 a black point indicates each studied locality where tardigrades occur; each black point is numbered with the number assigned to each locality in Table III. It seems that there is a major concentration of points in Portugal, specially in the Northwest (Fig. 1), while in Spain the points are sparse, but become a bit denser in the Pyrenees and in the centre of Spain (mainly in Madrid).

In Table IV we show world-wide taxonomic category information on tardigrades found in the studied area. Section A shows the number of classes, orders, families, genera and species in the entire World and in the Iberian Peninsula; also included percentage of world species found in the Iberian Peninsula for each taxonomic category. This section also displays the number of genera and species found in Portugal and in Spain, genera and species that occur only in Spain or in Portugal and percentages according to world-wide information. Section B, displays number and percentage of species per family and per genera according to world-wide information, in the Iberian Peninsula and in the rest of the world.

We can observe that the world-wide pattern is repeated in the Iberian Peninsula. The world's most diverse families (Table IV), in species and genus number, that are also found in the Iberian Peninsula, are the most diverse families there too (i.e., for Hypsibiidae, $43 \%$ of genera and $11 \%$ of species occur in the Iberian Peninsula; Table IV). With reference to species number in the generea, the world's most diverse genera, that are also found in the Iberian Peninsula are also the most diverse genera there (Table IV).

Table V displays $p$ values and Spearman correlation coefficients and Table VI $p$ values from Kruskal-Wallis analysis and indicates the variables with the highest values for continuous variables analyzed. Two parallel analysis have been made, one with data collected from all habitat-substratums sampled in the Iberian Peninsula where tardigrades were found (Tables V and VIA), and another analysis with data only from mosses (Tables $\mathrm{V}$ and VIB), collected uniformly in points sampled in the Iberian Peninsula. Spearman correlation coefficients are qualitatively equal (always pisitive correlations and similar magnitudes), but vaules from analysis with mosses only are lower than those carried out over all habitats-substratums.

New records found in our samplings in the Iberian Peninsula are presented in Table II, which specifies class, order, family and genus of each species found. Synonymies found for each new species, identification number that corresponds with the numerical order on Table III and Figure 1 (new localities sampled in Iberian Peninsula, mainly from centre of Spain, are marked on Figure 1 with an "X"), UTM coordinates, habitat-substratums analyzed and number of specimens found for each species. New records for the Iberian Peninsula are: Dactylobiotus parthenogeneticus, Dactylobiotus selenicus, Murrayon dianeae, Diphascon (Diphascon) nodulosum (first record in Europe), Diphascon (Diphascon) pingue and Isohypsibius marcellinoi. These results increase the number of Tardigrada phylum species found in the Iberian Peninsula to $118,5 \%$ more than the specific diversity previously known in the Iberian Peninsula.

There are broadly distributed limnoterrestrial species (large number of registers per species involving a large number of habitat-substratums and localities; Table V), appearing in Portugal and Spain in a large number of localities (Table II and III, Fig. 1), such as Macrobiotus hufelandi (130 localities) or Milnesium tardigradum (53 localities). The majority of these species are considered to be cosmopolitan by McInnes, in the revision made in 1994. However, there are other species with a more restricted distribution, appearing only in one locality (Table II and III; Fig. 1). The majority of species that are more or less widely distributed in the Iberian Peninsula appear in many of the habitat-substratums studied (i. e., Macrobiotus hufelandi) (Table II) with a high correlation coefficient $(+0.81$; Table $\mathrm{V})$; but species that occur in many of the habitat-substratums studied do not 
Table IV.- (A) Absolute number and percentage of limnoterrestrial species with respect to world-wide distribution of each Tardigrada taxonomy category in the World and in the Iberian Peninsula (IP). $*=$ percentage with respect to world information; $+=$ percentage with respect to Iberian Peninsula information; \# = genus and species that appear only in Spain or in Portugal. (B) In the first part of the Table (left), number (No) and percentage (\%) of limnoterrestrial genus and species (spp) with respect to World information in each of Tardigrada families found in the Iberian Peninsula (IP). In second part of the Table (right), number (No) and percentage (\%) of limnoterrestrial species (spp) with respect to World information in each of the Tardigrada genus found in the Iberian Peninsula.

Tabla IV.- (A) Número absoluto y porcentaje de especies limnoterrestres respecto a la distribución mundial de cada categoría taxonómica del phylum Tardigrada en el Mundo y en la Península Ibérica (IP). * = porcentaje respecto a la información mundial; $+=$ porcentaje respecto a la información peninsular; \# = géneros y especies que aparecen sólo en España o en Portugal. (B) En la primera parte de la Tabla (izquierda), número (No) y porcentaje (\%) de géneros y especies (spp) limnoterrestres respecto a la información mundial de cada familia del phylum Tardigrada encontrada en la Península Ibérica (IP). En la segunda parte de la Tabla (derecha), número (No) y porcentaje (\%) de especies limnoterrestres recpecto a la información mundial de cada género del phylum encontrado en la Península Ibérica.

(A)

\begin{tabular}{lccccccc}
\hline $\begin{array}{l}\text { Taxonomic } \\
\text { categories } \downarrow\end{array}$ & \multicolumn{2}{c}{ Absolute number of each taxonomic category } & \multicolumn{3}{c}{ Percentage of species (\%) } \\
& World & IP & Spain & Portugal & IP* & Spain+ & Portugal+ \\
\hline Class & 3 & 2 & 2 & 2 & 66.7 & 100 & 100 \\
Order & 5 & 3 & 3 & 3 & 60.0 & 100 & 100 \\
Family & 20 & 5 & 5 & 5 & 25.0 & 100 & 100 \\
Genus & 57 & 23 & $19(4 \#)$ & $18(4 \#)$ & 40.4 & $19.8(4.2 \#)$ & $18.8(4.2 \#)$ \\
Species & 783 & 92 & $72(35 \#)$ & $59(23 \#)$ & 11.7 & $78.3(38.0 \#)$ & $57.4(24.0 \#)$ \\
\hline
\end{tabular}

(B)

\begin{tabular}{|c|c|c|c|c|c|c|c|c|c|c|}
\hline \multirow{4}{*}{ Families $\downarrow$} & \multicolumn{6}{|c|}{ Genus and Species per Family } & \multicolumn{4}{|c|}{ Species per Genus } \\
\hline & \multicolumn{2}{|c|}{ World (Number) } & \multicolumn{4}{|c|}{ Iberian Peninsula } & \multirow{3}{*}{ Genus $\downarrow$} & \multirow{3}{*}{$\begin{array}{l}\text { World } \\
\text { No }\end{array}$} & \multirow{3}{*}{$\begin{array}{l}\text { IP } \\
\text { No }\end{array}$} & \multirow[b]{3}{*}{$\%$} \\
\hline & Genus / Family & Species /Family & Genus & Family & Spp & Family & & & & \\
\hline & & & No & $\%$ & No & $\%$ & & & & \\
\hline Milnesiidae & 2 & 7 & 1 & 50.0 & 1 & 14.3 & Milnesium & 1 & 1 & 100.0 \\
\hline Calohypsibiidae & 4 & 16 & 1 & 25.0 & 3 & 18.8 & Calohypsibius & 4 & 3 & 75.0 \\
\hline Hypsibiidae & 21 & 295 & 9 & 42.9 & 32 & 10.8 & $\begin{array}{l}\text { Astatumen } \\
\text { Diphascon } \\
\text { Doryphoribius } \\
\text { Hebesuncus } \\
\text { Hypsibius } \\
\text { Isohyosibius } \\
\text { Itaquascon } \\
\text { Pseudobiotus } \\
\text { Ramazzottius }\end{array}$ & $\begin{array}{r}5 \\
77 \\
16 \\
3 \\
39 \\
117 \\
7 \\
4 \\
18\end{array}$ & $\begin{array}{l}1 \\
9 \\
2 \\
1 \\
7 \\
9 \\
1 \\
1 \\
1 \\
2\end{array}$ & $\begin{array}{r}20.0 \\
11.7 \\
12.5 \\
33.3 \\
18.0 \\
7.6 \\
14.3 \\
25.0 \\
11.1\end{array}$ \\
\hline Macrobiotidae & 11 & 197 & 5 & 45.5 & 25 & 12.7 & $\begin{array}{l}\text { Dactylobiotus } \\
\text { Macrobiotus } \\
\text { Minibiotus } \\
\text { Murrayon } \\
\text { Richtersius }\end{array}$ & $\begin{array}{r}10 \\
142 \\
23 \\
7 \\
1\end{array}$ & $\begin{array}{r}3 \\
17 \\
3 \\
2 \\
1\end{array}$ & $\begin{array}{r}30.0 \\
12.0 \\
13.0 \\
28.6 \\
100.0\end{array}$ \\
\hline Echiniscidae & 12 & 235 & 7 & 58.3 & 32 & 13.6 & $\begin{array}{l}\text { Bryodelphax } \\
\text { Cornechiniscus } \\
\text { Echiniscus } \\
\text { Hypechiniscus } \\
\text { Parechiniscus } \\
\text { Pseudechiniscus } \\
\text { Testechiniscus }\end{array}$ & $\begin{array}{r}11 \\
9 \\
151 \\
6 \\
2 \\
35 \\
6\end{array}$ & $\begin{array}{r}3 \\
2 \\
16 \\
1 \\
2 \\
5 \\
1\end{array}$ & $\begin{array}{r}27.3 \\
22.2 \\
10.6 \\
16.7 \\
100.0 \\
14.3 \\
16.7\end{array}$ \\
\hline
\end{tabular}


Table V.- Spearman correlation analysis results. Spearman coefficient $(\mathrm{R})$ and $\mathrm{p}$ values from significant correlation analysis between pairs of variables and per species, genus, habitats and localities specified in each case. No $=$ number, HS $=$ habitat-substratums.

Tabla V.- Resultados del análisis de correlación de Spearman. Coeficiente de Spearman (R) y valores de p para los análisis de correlación significativos entre pares de variables y por especie, género, hábitat y localidad especificado en cada caso. No = número, $\mathrm{HS}$ = habitat-sustrato.

\begin{tabular}{lllll}
\hline & \multicolumn{2}{l}{ All Habitat-Substratums } & \multicolumn{2}{l}{ Only with Mosses } \\
Pairs of Variables analyzed & p value & R & p value & R \\
\hline No Species with No Registers per locality & 0.00 & +0.95 & 0.00 & +0.93 \\
No Species with No HS per locality & 0.00000 & +0.38 & 0.000007 & +0.33 \\
No Species with No Localities per region & 0.00033 & +0.52 & 0.039 & +0.37 \\
No Species with No Register per region & 0.000000 & +0.94 & 0.000000 & +0.90 \\
\hline No Genus with No Registers per locality & 0.000000 & +0.87 & 0.000000 & +0.84 \\
No Genus with No HS per locality & 0.000000 & +0.43 & 0.000000 & +0.40 \\
No Genus with No Localities per region & 0.000003 & +0.65 & 0.023 & +0.40 \\
No Genus with No Register per region & 0.000000 & +0.89 & 0.000000 & +0.79 \\
\hline No HS with No Register per species & 0.000000 & +0.81 & 0.000000 & +0.69 \\
No HS with No Register per locality & 0.000000 & +0.52 & 0.000000 & +0.48 \\
No HS with No Localities per region & 0.000077 & +0.57 & 0.0011 & +0.55 \\
\hline No Localities with No HS per species & 0.000000 & +0.81 & 0.000000 & +0.69 \\
No Localities with No Registers per HS & 0.000000 & +0.98 & 0.000000 & +0.98 \\
No Localities with No Registers per region & 0.000000 & +0.90 & 0.000000 & +0.85 \\
\hline No Registers with No Localities per species & 0.00 & +0.98 & 0.00 & +0.93 \\
\hline
\end{tabular}

have to be widely distributed in the Iberian Peninsula (i.e., Macrobiotus harsmworthi is not widely distributed in the Iberian Peninsula but occurs in the majority of habitats sampled in the Iberian Peninsula; Table II).

Mosses plus lichens are the habitats with the highest tardigrade species specific and genera diversity (Table II, VIA and B), but the greatest extensive sampling effort (number of localities) have been made too in these habitats (Tables VIA and B). Similar situation is in leaf-litter (Table VIA). While considerable sampling effort has been directed towards certain habitats (such as mosses; Table VIB), others have been greatly overlooked and a lot of habitats in the Iberian Peninsula where tardigrades can live have been not sampled at all (i.e., interstitial habitats in caves; Table II) or else insufficiently (marine habitats that have high species diversity without a great sampling effort associated), as demonstrated by new sampling (Table III). These habitats seem to have new tardigrade species to add to the previously known Iberian Tardigrada diversity (Table II). While there are species restricted to few habitat-substratums, this does not mean that a specific species-habitat relationship exists. It could be that in some localities few or rare habitat-substratums were sampled and the rela- tionship would then be between locality-species instead of habitat-species. To verify the existence of a true species-habitat relationship, further studies are required.

With reference to localities, of the few (Tables II and III) populated by 10 or more species, one is in Andorra (number of locality: 29), one in Spain (13) and 7 in Portugal $(43,25,139,40,9,107,114)$, with the majority in Northwest Portugal (Fig. 1). These localities are characterised by the highest number of registers and number of sampled habitatsubstratums (Table V). The results for genera are the same (Table V). The greatest sampling effort has been made in Portugal, both in locality number (55.3\% from total) and in register number $(68.1 \%$ from a total of 1020 registers) (Table III). Nevertheless, the highest percentages of limnoterrestrial species and genera in the Iberian Peninsula were found in Spain $(78.3 \%$ and $82.6 \%$, respectively) (Table II).

\section{Discussion}

Articles about Iberian tardigrade studies have appeared irregularly, and do not lend themselves to a global statistical analysis due to bias in geograp- 
Table VI.- Kruskal-Wallis analysis results from analysis of all habitat (A) and only of mosses (B): p values (significant) and greatest values for type of habitat-substratums; the first column is the continuous variable analysed with the type of habitat substratum (discrete variable). $\mathrm{No}=$ number, $\mathrm{HS}=$ habitat-substratums, $\mathrm{AM}=$ marine algae, $\mathrm{H}=$ leaf-litter, $\mathrm{IM}=$ interstitial marine habitats, $\mathrm{M}=$ moss, $\mathrm{MA}=$ moss from trunks, $\mathrm{MDulc}=$ moss from freshwater, $\mathrm{ME}=$ moss from soil, $\mathrm{ML}=$ moss + lichens, $\mathrm{MR}=$ moss from rocks, $\mathrm{MLA}=$ moss + lichens from trunks, $\mathrm{MLR}=$ moss + lichens from rocks, $\mathrm{MLHep}=$ moss + lichens + hepatics, MLHEPDV= moss + lichens + hepatics + detritus, $M L H e p A=$ moss + lichens + hepatics from trunks, MLHepR $=$ moss + lichens + hepatics from rocks, $\mathrm{SM}=$ marine sediment, $\mathrm{TUR}=$ peatland.

Tabla VI.- Resultados del análisis de Kruskal-Wallis analysis para el análisis con todos los hábitats (A) y sólo con musgo (B): valores de $\mathrm{p}$ (significativos) y qué hábitats-sustratos tiene los valores más altos; la primera columna es la variable contínua analizada con el tipo de hábitat sustrato (variable discreta). $\mathrm{No}=$ número, $\mathrm{HS}=$ hábitat-sustrato, $\mathrm{AM}=$ alga marina, $\mathrm{H}=$ hojarasca, $\mathrm{IM}=$ hábitat intersticial marino, $\mathrm{M}=$ musgo, $\mathrm{MA}=$ musgo arborícola, $\mathrm{MDulc}=$ musgo dulceacuícola, $\mathrm{ME}=$ musgo edáfico, $\mathrm{ML}=$ musgo+líquen, $\mathrm{MR}=$ musgo rupícola, $\mathrm{MLA}=$ musgo+líquen arborícola, $\mathrm{MLR}=$ musgo+líquen rupícola, $\mathrm{MLHep=}$ musgo+líquen+hepática, MLHEPDV= musgo+líquen+hepática+detritus, MLHepA $=$ musgo+líquen+hepática arborícola, $\mathrm{MLHepR}=$ musgo+líquen + hepática rupícola, $\mathrm{SM}=$ sedimentos marinos, $\mathrm{TUR}=$ turbera.

\begin{tabular}{lll}
\cline { 2 - 3 } (A) & ALL HABITAT-SUBSTRATUMS \\
\hline Continuous variables & $\mathbf{p}$ & Biggest values for continuos variables \\
\hline No Species per region & 0.007 & ML $>$ SM $>H>M>M L A$ \\
No Genus per region & 0.004 & ML $>\mathrm{H}>\mathrm{MLA}>\mathrm{M}>\mathrm{MLR}>\mathrm{SM}>\mathrm{MA}$ \\
No Localities per region & 0.001 & $\mathrm{ML}>\mathrm{H}>\mathrm{ML}>\mathrm{M}>\mathrm{MLHEPA}$ \\
\hline
\end{tabular}

\begin{tabular}{|c|c|c|}
\hline (B) & $\overline{\text { ONLY WITH MOSSES }}$ & \\
\hline Continuous variables & $\mathbf{p}$ & Biggest values for continuos variables \\
\hline No Species per region & 0.006 & ML $>$ MLA $>$ M $>$ MLR $>$ MR $>$ MA $>$ ME $>$ MLHEPR $>$ MLHEPA \\
\hline No Genus per region & 0.006 & $\mathrm{ML}>\mathrm{MLA}>\mathrm{M}>\mathrm{MLR}>\mathrm{MA}>\mathrm{MR}>\mathrm{MDULC}$ \\
\hline No Localities per region & 0.019 & $\mathrm{ML}>\mathrm{M}=\mathrm{MLHEPA}=\mathrm{ME}>\mathrm{MLA}>\mathrm{MLHEPR}>\mathrm{MR}>\mathrm{MLR}>\mathrm{MA}$ \\
\hline
\end{tabular}

hical location of sampling points and in the analyzed habitats. Anyway, increasing sampling effort (extensively (in number of localities), intensively (in number of registers) or in habitats sampled) more specific and generic diversity has been found in the Iberian Peninsula (Table V).

It seems that the species diversity of some habitats may be greater than others; i.e., moss and lichen assemblages seem to be the most diverse habitats (Table II), thought they are also the most sampled (in $55.2 \%$ of the localities). When the Iberian Peninsula is considered as a geographic whole it becomes evident that major differences in habitat sampling are due more to sampling behaviour than to absence of habitat (mosses, lichens or soil are found almost anywhere in the Iberian Peninsula). Future research could thus focus on the relationship between tardigrade diversity and habitat type.

There are 1.2 times more species found in Spain than in Portugal, but there are 1.3 times more localities sampled in Portugal than in Spain. Species diversity of Tardigrada phylum seems greater in Spain than in Portugal, but this situation should be studied further. Species diversity may be due to the greater number of habitats sampled in Spain (for instance, there are no works on Portuguese marine tardigrades). The main geographic difference between the two countries is their surface areas, the Spanish surface area is 5.5 times larger than the Portuguese $\left(89,743 \mathrm{~km}^{2}\right.$ vs. $\left.504,750 \mathrm{~km}^{2}\right)$. On the other hand, Andorra, with a surface area of $468 \mathrm{~km}^{2}$, has the highest species diversity in relation with its surface area: 11 tardigrade species from 7 genera from 2 references (see Table III). Sampling effort should be directed towards increasing habitat diversity sampled, because its extencion seems to be positively related with species and genus diversity (Table V). Also the geographic areas of study should be increased to determine which species are cosmopolitan and to verify if Iberian endemisms exist.

If the aims previously indicated will be achieved, Spanish Tardigrada phylum studies will ideally be, one day, as complete as those conducted in Portugal, both in the extension and intensity of the sampling. As it seens in results obtained in this work, tardigrade species diversity in Spain is found to be high. The great variety and diversity of 
Spanish landscapes and habitats along with its unique geological history could favour the discovery of new species to science. The greater species diversity would then be of interest to a further approach to systematics and conservation.

\section{ACKNOWLEDGEMENTS}

The author gratefully acknowledges the support of this research provided by the Comunidad de Madrid, Consejo Superior de Investigaciones Científicas (CSIC) and Museo Nacional de Ciencias Naturales of Madrid (CSIC). I also thank Dr. A. I. Camacho, whose critical comments significantly contributed to this work, and to Prof. R. Bertolani and Dr. R. Guidetti who greatly helped with taxonomical problems.

\section{References}

Barros, R. \& Da CunHA, A. X., 1937. Liste de quelques Tardigrades de Coïmbre. Comptes Rendus du XII Congres International de Zoologie”, vol. III. Lisboa.

Crowe, J. H., 1975. The physiology of criptobiosis in tardigrades. Memoirs dell'Istituto Italiano di Idrobiologia, 32 suppl.: 37-59.

Crowe, J. H. \& Crowe, L. M., 2000. Preservation of mammalian cells learning nature's tricks. Nature Biotechnology, 18(2):145-146.

Da Cunha, A. X., 1941. Tardigrados da Fauna Portuguesa. Memorias e Estudos do Museu Zoologico da Universidade da Coimbra, 120: 1-25.

Da CunHA, A. X., 1943. Un Tardigrade nouveau du Portugal: Hypsibius placophorus sp. nov. Memorias e Estudos do Museu Zoologico da Universidade da Coimbra, 143: 1-3.

Da Cunha, A. X., 1944a. Tardigrados do Fauna Portuguesa II. Memorias e Estudos do Museu Zoologico da Universidade da Coimbra, 15: 1-11.

DA CunHA, A. X., 1944b. Echiniscus multispinosus sp. n., un Tardigrade nouveau de la Faune Portugaise. Memorias e Estudos do Museu Zoologico da Universidade da Coimbra, 159: 1-5.

Da Cunha, A. X., 1947a. Tardigrados do Fauna Portuguesa III. Memorias e Estudos do Museu Zoologico da Universidade da Coimbra, 177: 1-8.

Da Cunha, A. X., 1947b. Description d'un Tardigrade nouveau de la Faune Portogaise: Parechiniscus unispinosus sp. n. Memorias e Estudos do Museu Zoologico da Universidade da Coimbra, 180: 1-5.

DA Cunha, A. X., 1948. Tardigrados do Fauna Portuguesa IV. Memorias e Estudos do Museu Zoologico da Universidade da Coimbra, 188: 1-5.

Fontoura, A. P., 1981. Contribution pour l'étude des tardigrades terrestres du Portugal avec la description d'une nouvelle espèce du genre Macrobiotus.
Publicacoes do Instituto de Zoologia 'Dr. Augusto Nobre'. Facultad de Ciencias do Porto, 160: 1-24.

FontourA, A. P., 1982. Deux nouvelles espèces de Tardigrades muscicoles du Portugal. Publicaçoes do Instituto de Zoologia 'Dr. Augusto Nobre'. Facultad de Ciencias do Porto, 165: 5-19.

Garey, J.R., Krotec, M., Nelson, D. R. \& Brooks, J., 1996. Molecular analysis supports a tardigrade-arthropod association. Invertebrate Biology, 115(19): 79-88.

Garey, J.R., Nelson, D. R., Mackey, L. J. \& Li, J., 1999. Tardigrade phylogeny: congruency of morphological and molecular evidence. Zoologischer Anzeiger, 238: 205-210.

Giribet, G., Carranza, S., Baguna, J., Rintort, M. \& RIBERA, C., 1996. First molecular evidence for the existence of a Tardigrada + Arthropoda clade. Molecular Biology and Evolution, 13(1): 76-84.

Goeze, J. A. E., 1773. Uber den Kleinen Wasserbär. In: H. K. Bonnet (ed.). Abhandlungen aus der Insectologie, Ubers. Usw, 2. Beobachtg.

Hartvingsen, G., 2001. Evolution and Biodiversity. In: S. A. Levin (ed.). Enciclopedia of Diversity. San Diego. Academic Press: 393-401.

HeInIS, F., 1908. Beitrag zur Kenntnis der Moosfauna der kanarischen Inseln. Zoologischer Anzeiger, XXXIII: 711.

Humphries, C. J., Williams, P. H. \& VAne-Wright, R. I., 1995. Measuring biodiversity value for conservation. Annual Review of Ecology and Systematics, 26: 93-111.

KIESER, J. A., 1993. Evolution, developmental instability and the theory of acquisition. Genetica, 89: 219-225.

Kristensen, R. M. \& Hallas, T. E., 1980. The tidal genus Echiniscoides and its variability, with erection of Echiniscoidea fam. n. Zoological Scripta, 9(2): 113-127.

LEwIN OsORIO, M., 1984. Nota sobre los tardígrados muscícolas de Cataluña. Publicaciones del Departamento de Zoología (Barcelona), 10: 39-49.

MAdrid Moreno, J., 1911. Datos para el estudio de plankton del río Lozoya. Boletín de la Real Sociedad Española de Historia Natural, XI: 173.

MAucci, W., 1979. Osservazioni sul valore tassonomico di Macrobiotus recens Cuénot, 1932 (Tardigrada, Macrobiotidae). Natura, Milano, 70(4): 258-264.

MauccI, W., 1983. Echiniscus bisculptus n. sp. del Marocco, ed Echiniscus lichenorum n. sp., del Portogallo (Tardigrada, Echiniscidae). Atti della Societá Italiana di Scienze Naturale e del Museo Civico di Storia Naturale Milano, 124(3/4): 257-261.

MaUcCI, W., 1991. Due nuove specie di Tardigradi muscicoli della Spagna. Bollettino del Museo Civico di Storia Naturale di Verona, 15: 257-264.

Maucci, W. \& Durante Pasa, M. V., 1984. Tardigradi della Penisola Iberica. Miscellania Zoologica, 8: 67-80. 
MaUCCI, W. \& DuRAnTe PaSA, M. V., 1985. Il Tardigradi della fauna Portoghese. Anais da Facultade de Ciencias, Universidade do Porto, 65(1/4): 127-174.

Maucci, W. \& Ramazzotti, G., 1981. Pseudechiniscus gruppo cornutus, con descrizione di una nuova specie (Tardigrada, Echiniscidae). Memorie dell'Istituto Italiano di Idrobiologia Dott. Marco de Marchi, 39: 147-151.

MCInNeS, S. J., 1991. Notes on tardigrades from the Pyrenees, including one new species. Pedobiologia, 35(1): 11-26.

MCINNES, S. J., 1994. Zoogeographic distribution of terrestrial and freshwater tardigrades from current literature. Journal of Natural History, 28: 257-352.

MinelCiC, F., 1954. Contribución al conocimiento de los tardígrados en España; estudio sistemático-ecológico. Anales de Edafología y Fisiología Vegetal, 13: 103-109.

MinelCiC, F., 1955. Zweineue Tardigradenarten aus Spaien. Zoologischer Anzeiger, 155(11/12): 309-311.

Nelson, D. R., 1982. Developmental biology of the Tardigrada. En: F. Harrison \& R. Cowden (eds.). Developmental biology of freshwater Invertebrates. Alan R. Liss. New York: 363-398.

Nelson, D. R., 1995. The hundred-year hibernation of the waterbear. Natural History, 84(3): 62-65.

Nelson, D. \& Marley, N. J., 2000. The biology and ecology of lotic Tardigrada. Freshwater Biology, 44(1): 93-108.

Nielsen, C., 1995. Animal evolution. Interrelationship of the living phyla. Oxford University Press. Oxford. $175 \mathrm{pp}$.

Pardo García, L., 1919. Notas preliminares sobre el plankton de Onteniente (Valencia). Boletín de la Real Sociedad Española de Historia Natural, Acta de la Sección de Valencia, XIX(6): 289-293.

PARdo García, L., 1921. Notas preliminares sobre el plankton de Onteniente (Valencia). Asociación Española para el progreso de las ciencias, Congreso de Oporto, VI: 215-220

RamazzotTI, G., 1972. Il phylum Tardigrada. II edizione riveduta aggiornata. Memorie dell'Istituto Italiano di Idrobiologia, 28: 1-732.

Ramazzotti, G. \& Maucci, W., 1983. Il phylum Tardigrada. III edizione riveduta e aggiornata. Memorie dell'Istituto Italiano di Idrobiologia, 41: $1-1012$.
RodríGuez Roda, J., 1946. Contribución al estudio de los Tardígrados de España. Nota previa sobre los de Aralar. In: R. Margalef (ed.). Aportación al estudio de la fauna y flora vasco-navarra, Sierra de Aralar. CSIC. Estación de Estudios Pirenaicos. Zaragoza: 67-82.

Rodríguez RodA, J., 1947. Contribución al estudio de los tardígrados de España. Publicaciones del Instituto de Biología Aplicada, 2: 111-115.

RodríGuez RodA, J., 1949. Tardígrados del centro de España. Publicaciones del Instituto de Biología Aplicada, 6: 27-40.

RodríGUEz Roda, J., 1951. Algunos datos sobre la distribución de los tardígrados españoles. Boletín de la Real Sociedad Española de Historia Natural, 49: 7583.

RodríGuez Roda, J., 1952. Tardígrados de la Fauna Española. Trabajos del Museo de Ciencias Naturales de Barcelona, Nueva Serie Zoológica, 1(4): 1-84.

Swingland, I. R., 2001. Definition of Biodiversity. In: S. A. Levin (ed.). Enciclopedia of Diversity. San Diego. Academic Press: 377-391.

Villora Moreno, S. \& De Zio Grimaldi, S., 1993. Redescription and ecology of Batillipes phreaticus Renaud-Debyser, 1959 (Arthrotardigrada, Batillipedidae) in the gulf of Valencia (western Mediterranean). Cahiers de Biologie Marine, 34(3): 387-399.

Villora Moreno, S. \& De Zio Grimaldi, S., 1996. New record of marine Tardigrada in the Mediterranean sea. Zoological Journal of the Linnean Society, 116(1-2): 149-166.
Recibido, el 5-XI-2002 Aceptado, el 17-XII-2002 Publicado, el 31-XII-2002 